\title{
Singlino-dominated dark matter in general NMSSM
}

\author{
Junjie Cao, Demin Li, Jingwei Lian, Yuanfang Yue and Haijing Zhou \\ Department of Physics, Henan Normal University, \\ Xinxiang 453007, China \\ E-mail: junjiec@alumni.itp.ac.cn, deminli@foxmail.com, \\ ljwfly@hotmail.com, yuanfang405@gmail.com, zhouhaijing0622@163.com
}

AbStRact: The general Next-to-Minimal Supersymmetric Standard Model (NMSSM) describes the singlino-dominated dark-matter (DM) property by four independent parameters: singlet-doublet Higgs coupling coefficient $\lambda$, Higgsino mass $\mu_{\text {tot }}$, DM mass $m_{\tilde{\chi}_{1}^{0}}$, and singlet Higgs self-coupling coefficient $\kappa$. The first three parameters strongly influence the DM-nucleon scattering rate, while $\kappa$ usually affects the scattering only slightly. This characteristic implies that singlet-dominated particles may form a secluded DM sector. Under such a theoretical structure, the DM achieves the correct abundance by annihilating into a pair of singlet-dominated Higgs bosons by adjusting $\kappa$ 's value. Its scattering with nucleons is suppressed when $\lambda v / \mu_{\text {tot }}$ is small. This speculation is verified by sophisticated scanning of the theory's parameter space with various experiment constraints considered. In addition, the Bayesian evidence of the general NMSSM and that of $Z_{3}$-NMSSM is computed. It is found that, at the cost of introducing one additional parameter, the former is approximately $3.3 \times 10^{3}$ times the latter. This result corresponds to Jeffrey's scale of 8.05 and implies that the considered experiments strongly prefer the general NMSSM to the $Z_{3}$-NMSSM.

KEYWords: Supersymmetry Phenomenology

ARXIV EPRINT: 2102.05317 


\section{Contents}

1 Introduction 1

2 Theoretical preliminaries $\quad 3$

2.1 General NMSSM 3

2.2 DM relic density 8

$\begin{array}{lll}2.3 & \text { DM-nucleon scattering cross-sections } & 10\end{array}$

3 Numerical results $\quad 11$

$\begin{array}{lll}3.1 & \text { Research strategy } & 12\end{array}$

$\begin{array}{lll}3.2 & \text { Posterior probability density } & 13\end{array}$

$\begin{array}{ll}3.3 & \text { Profile likelihood of DM physics } \\ \end{array}$

$\begin{array}{ll}\text { 3.4 Constraints from LHC search for electroweakinos } & 17\end{array}$

4 Conclusions $\quad 20$

\section{Introduction}

With the smooth development of various dark-matter (DM) detection experiments in the past decades, DM research has gradually become a hot topic. Massive Weakly Interacting Particles (WIMPs) are considered the most promising DM candidates because they can naturally predict the abundance measured by the Planck experiment in $[1,2]$. This phenomenon is called the WIMP Miracle in the literature [3, 4]. One distinctive feature of the candidates is that the spin-independent (SI) cross-section of their scattering with nucleons is approximately $10^{-45} \mathrm{~cm}^{2}$ since they are usually supposed to couple to the Standard Model (SM) particles through weak interactions [5]. Nevertheless, the DM direct detection experiments give a different answer; that is, so far, the XENON-1T and PandaX-II experiments have restricted the cross-section below the order of $10^{-46} \mathrm{~cm}^{2}[6-8]$, which implies that the interaction between DM and nucleons is at most feeble [9]. This fact reveals a general conclusion that simple WIMP theories face significant experimental challenges.

In popular supersymmetric theories, the lightest neutralino, $\tilde{\chi}_{1}^{0}$, as the lightest supersymmetric particle (LSP), is a stable WIMP in theories' natural parameter space and usually acts as a DM candidate. It affects both the signal of supersymmetric particles at colliders and the evolution of the universe, so it has been the focus of DM physics over the past several decades [3]. With the increasing sensitivity of DM direct detection experiments, this candidate is becoming increasingly more difficult to be consistent with both the detection experiments and the abundance naturally [9]. Taking the Minimal Supersymmetric Standard Model (MSSM) as an example, the neutralino with $m_{\tilde{\chi}_{1}^{0}} \lesssim 1 \mathrm{TeV}$ must be Bino-dominated in its component to produce correct relic abundance. In this case, the 
SI and spin-dependent (SD) cross-sections rely on Higgsino mass in different ways. If the Higgsinos are moderately light, at least one of them would be considerable in size [10]. As a result, the Higgsinos must be heavier than approximately $300 \mathrm{GeV}$ after considering current XENON-1T experimental constraints on the cross-sections and approximately $600 \mathrm{GeV}$ supposing no DM signal detected in the future LZ experiments [10]. Additionally, a global fit of the MSSM to various experimental data known in 2017 preferred the Higgsinos to be heavier than approximately $350 \mathrm{GeV}$ at a $95 \%$ confidence level [11]. This conclusion should be significantly improved since both DM detection experiments and the LHC search for supersymmetry have progressed rapidly in recent years. These facts reveal that the theory already has undergone a relatively significant fine-tuning to predict $Z$ boson mass. With further development of DM detection experiments, the tuning will become even more significant.

The dilemma of the MSSM inspired our study of the Next-to-Minimal Supersymmetric Standard Model (NMSSM) [12], which extends the MSSM by one gauge-singlet superfield and is another popular realization of supersymmetry, and to consider a Singlino-dominated neutralino as a DM candidate. The theory introduces the inputs $\lambda$ and $\kappa$ to parameterize singlet-doublet Higgs interactions and singlet Higgs self-interactions. Thus, the couplings between the DM and nucleus depend on the Higgsino composition of the DM, which, for fixed DM mass, is determined by the dimensionless parameter $\lambda$ and the Higgsino mass $\mu_{\text {tot }}$ (see discussions in this paper). When $\lambda \gtrsim 0.3$ and $\mu_{\text {tot }} \lesssim 500 \mathrm{GeV}$, the scattering crosssections of the DM and nucleus tend to be too large to be consistent with the XENON-1T constraints under the premise of the correct DM abundance [13]. This situation remains valid for any DM mass when the constraints from the LHC search for supersymmetry are included [9, 14], and it has a great impact on DM abundance [10]. For example, the annihilations $\tilde{\chi}_{1}^{0} \tilde{\chi}_{1}^{0} \rightarrow t \bar{t}, h A_{s}, h_{s} A_{s}$ were believed to play crucial roles in determining the abundance [5, 15], where $t, h, h_{s}$, and $A_{s}$ denote the top quark, SM-like Higgs boson, and singlet-dominated CP-even and CP-odd Higgs bosons, respectively. After considering the XENON-1T constraints, none of them, however, can be fully responsible for the correct abundance. Instead, DM prefered to co-annihilate with the Higgsinos to obtain the density, which corresponds to a correlated parameter space of $2|\kappa| \simeq \lambda$ with $\lambda \lesssim 0.1$ [9, 14]. It is worth adding that, with the improvement of the experimental sensitivity in detecting DM, the small upper bound of $\lambda$ will be further reduced.

It is noted that the above conclusions about the Singlino-dominated DM are established in the NMSSM with a $Z_{3}$ symmetry. In such a framework, to ensure that the neutralino is the LSP, $|\kappa|$ must be less than $\lambda / 2$ [12]. Consequently, it should be small after considering the DM experiments' substantial limitation on $\lambda$. This conclusion, however, does not hold in the general NMSSM (GNMSSM) due to the emergence of $Z_{3}$-violating terms. Specifically, the ratio of Singlino and Higgsino masses is no longer $2|\kappa| / \lambda$, so $|\kappa|$ may be much larger than $\lambda$ to predict the Singlino-dominated neutralino as the LSP. The Singlino-Higgsino complex system has four independent parameters, which may take $\lambda, \mu_{\text {tot }}, m_{\tilde{\chi}_{1}^{0}}$, and $\kappa$. This characteristic contrasts with that of the $Z_{3}$-NMSSM, which only contains three input parameters, i.e., $\lambda, \mu_{\text {tot }}$, and any one of $m_{\tilde{\chi}_{1}^{0}}$ and $\kappa$. An important application of these differences is that the singlet-dominated particles may form a secluded DM sector [16], 
which has the following salient features.

- Since the parameter $\kappa$ determines the interactions among the singlet-dominated particles, the Singlino-dominated DM can achieve the correct abundance by the process $\tilde{\chi}_{1}^{0} \tilde{\chi}_{1}^{0} \rightarrow h_{s} A_{s}$ through adjusting the value of $\kappa$.

- When the parameter $\lambda$ is small, the secluded sector communicates with the SM sector only through the weak singlet-doublet Higgs mixing. In this case, the interaction between the DM and nucleus is naturally feeble.

It is emphasized that $\lambda$ and $\kappa$ may play different roles in the DM physics of the GNMSSM. Similar to the $Z_{3}$-NMSSM, the parameters $\lambda$, $\mu_{\text {tot }}$, and $m_{\tilde{\chi}_{1}^{0}}$ mainly affect the coupling between the DM and nucleons, so they have been strongly restricted by DM direct detection (DD) experiments. In contrast, the singlet fields' self-interactions can be entirely responsible for the DM density, where the parameter $\kappa$ plays a crucial role. Owing to this characteristic, the GNMSSM has a broad parameter space consistent with the current DM experimental results. This fact has been neglected in the literature. Considering the dilemma of the $Z_{3}$-NMSSM in DM physics, the DM physics of the GNMSSM are scrutinized herein. Our results are quite different from those of previous studies on Singlino-dominated DM, which worked in the framework of the $Z_{3}$-NMSSM $[9,10,13,14,17-23]$.

The rest of this paper is organized as follows. First, the key features of the GNMSSM are introduced in section 2. In particular, an economic GNMSSM realization is considered. The research strategy and numerical results are described in section 3 to show the characteristics of DM physics. Finally, conclusions are drawn in section 4.

\section{Theoretical preliminaries}

In this section, the GNMSSM's basics are reviewed. The Singlino-dominated DM's properties will be elucidated in detail by analytic formulae for the case of massive-charge Higgs and gauginos.

\subsection{General NMSSM}

The GNMSSM augments the MSSM by a gauge singlet superfield $\hat{S}$ that does not carry any leptonic or baryonic number. Thus, its Higgs sector contains $\hat{S}$ and two $\mathrm{SU}(2)_{L}$ doublet superfields, $\hat{H}_{u}=\left(\hat{H}_{u}^{+}, \hat{H}_{u}^{0}\right)$ and $\hat{H}_{d}=\left(\hat{H}_{d}^{0}, \hat{H}_{d}^{-}\right)$. The general form of its superpotential is $[12]$

$$
W_{\mathrm{GNMSSM}}=W_{\text {Yukawa }}+\lambda \hat{S} \hat{H}_{u} \cdot \hat{H}_{d}+\frac{1}{3} \kappa \hat{S}^{3}+\mu \hat{H}_{u} \cdot \hat{H}_{d}+\frac{1}{2} \nu \hat{S}^{2}+\xi \hat{S},
$$

where $W_{\text {Yukawa }}$ is the Yukawa term for quarks and leptons, and $\lambda$ and $\kappa$ are dimensionless coefficients of the trilinear terms invariant under the $Z_{3}$ symmetry. The terms characterized by the bilinear mass parameters $\mu$ and $\nu$ and the singlet tadpole parameter $\xi$ break the $Z_{3}$ symmetry. Without the loss of generality, one can eliminate the linear term in $\hat{S}$ by displacing the field by a specific constant $c$, i.e., $\hat{S}=\hat{S}^{\prime}+c$, so that only the bilinear mass terms in $\hat{S}^{\prime}$ remain to violate the symmetry. As suggested by the studies in [24-27], the $\mu$ 
and $\nu$ 's natural smallness could stem from the breaking of an underlying discrete $\mathrm{R}$ symmetry, $Z_{4}^{R}$ or $Z_{8}^{R}$, at a low-energy scale. Additionally, it is noticeable that a non-minimal coupling $\chi$ of a Higgs bilinear to gravity was introduced to implement a superconformal symmetry in the GNMSSM [28, 29], and this coupling could drive inflation in the early universe [30]. In this case, the extra $\mu$-term is connected with $\chi$ via gravitino mass $m_{3 / 2}$, i.e., $\mu=\frac{3}{2} m_{3 / 2} \chi$. It is the only $\mathrm{Z}_{3}$-breaking term in superpotential due to the superconformal symmetry breaking at the Planck scale [31]. In this study, partly motivated by the inflation-inspired model ${ }^{1}$ and mainly for simplifying our analysis, a specific GNMSSM is investigated in which all dimensional parameters in the superpotential are zero except $\mu$, labeled as $\mu$-extended NMSSM ( $\mu$ NMSSM) hereafter. The superpotential and corresponding soft supersymmetry-breaking Lagrangian are reduced to the following forms:

$$
\begin{aligned}
W_{\mu \mathrm{NMSSM}}= & W_{\text {Yukawa }}+(\lambda \hat{S}+\mu) \hat{H}_{u} \cdot \hat{H}_{d}+\frac{1}{3} \kappa \hat{S}^{3}, \\
-\mathcal{L}_{\text {soft }}= & {\left[A_{\lambda} \lambda S H_{u} \cdot H_{d}+\frac{1}{3} A_{\kappa} \kappa S^{3}+B_{\mu} \mu H_{u} \cdot H_{d}+\text { h.c. }\right] } \\
& +m_{H_{u}}^{2}\left|H_{u}\right|^{2}+m_{H_{d}}^{2}\left|H_{d}\right|^{2}+m_{s}^{2}|S|^{2},
\end{aligned}
$$

where $H_{u}, H_{d}$, and $S$ denote the Higgs superfields' scalar components. Throughout this work, the $B_{\mu}$ term is set to be zero since it plays a minor role in this study and the supersymmetry-breaking mass parameters, $m_{H_{u}}^{2}, m_{H_{d}}^{2}$, and $m_{s}^{2}$, are fixed by solving the conditional equations to minimize the scalar potential. The Higgs sector is then described by parameters $\lambda, \kappa, A_{\lambda}, A_{\kappa}, \mu$, and the Higgs fields' vacuum expectation values (vevs) $\left\langle H_{u}^{0}\right\rangle=v_{u} / \sqrt{2},\left\langle H_{d}^{0}\right\rangle=v_{d} / \sqrt{2}$, and $\langle S\rangle=v_{s} / \sqrt{2}$ with $v=\sqrt{v_{u}^{2}+v_{d}^{2}} \simeq 246 \mathrm{GeV}$. The ratio of the vevs $\tan \beta \equiv v_{u} / v_{d}$ and $\mu_{\text {eff }} \equiv \lambda v_{s} / \sqrt{2}$ are chosen as input parameters.

Concerning the Higgs sector, it is convenient to work with the field combinations $H_{\mathrm{SM}} \equiv \sin \beta R e\left[H_{u}^{0}\right]+\cos \beta R e\left[H_{d}^{0}\right], H_{\mathrm{NSM}} \equiv \cos \beta R e\left[H_{u}^{0}\right]-\sin \beta R e\left[H_{d}^{0}\right]$, and $A_{\mathrm{NSM}} \equiv$ $\cos \beta \operatorname{Im}\left[H_{u}^{0}\right]-\sin \beta \operatorname{Im}\left[H_{d}^{0}\right][32,34]$, where $H_{\mathrm{SM}}$ represents the Higgs field in the SM with the vev $v / \sqrt{2}, H_{\mathrm{NSM}}$ denotes the other CP-even doublet Higgs field with a vanishing vev, and $A_{\mathrm{NSM}}$ corresponds to the CP-odd Higgs boson in the MSSM. In the bases $\left(H_{\mathrm{NSM}}\right.$, $\left.H_{\mathrm{SM}}, \operatorname{Re}[S]\right)$, the elements of the CP-even Higgs fields' squared mass matrix are formulated as [33]

$$
\begin{aligned}
& \mathcal{M}_{S, 11}^{2}=\frac{2 \mu_{\mathrm{eff}}\left(\lambda A_{\lambda}+\kappa \mu_{\mathrm{eff}}\right)}{\lambda \sin 2 \beta}+\frac{1}{2}\left(2 m_{Z}^{2}-\lambda^{2} v^{2}\right) \sin ^{2} 2 \beta, \\
& \mathcal{M}_{S, 12}^{2}=-\frac{1}{4}\left(2 m_{Z}^{2}-\lambda^{2} v^{2}\right) \sin 4 \beta, \\
& \mathcal{M}_{S, 13}^{2}=-\frac{1}{\sqrt{2}}\left(\lambda A_{\lambda}+2 \kappa \mu_{\mathrm{eff}}\right) v \cos 2 \beta, \\
& \mathcal{M}_{S, 22}^{2}=m_{Z}^{2} \cos ^{2} 2 \beta+\frac{1}{2} \lambda^{2} v^{2} \sin ^{2} 2 \beta,
\end{aligned}
$$

\footnotetext{
${ }^{1}$ It must be emphasized here that, contrary to studies in the inflation-inspired model [31, 33], we concern ourselves with the $\mu$ extension's generality irrespective of its origin, and therefore the inflation explanation and its corresponding gravitino DM scenario are not discussed.
} 


$$
\begin{aligned}
\mathcal{M}_{S, 23}^{2} & =\frac{v}{\sqrt{2}}\left[2 \lambda \mu_{\mathrm{eff}}+2 \lambda \mu-\left(\lambda A_{\lambda}+2 \kappa \mu_{\mathrm{eff}}\right) \sin 2 \beta\right], \\
\mathcal{M}_{S, 33}^{2} & =\frac{\lambda A_{\lambda} \sin 2 \beta}{4 \mu_{\mathrm{eff}}} \lambda v^{2}+\frac{\mu_{\mathrm{eff}}}{\lambda}\left(\kappa A_{\kappa}+\frac{4 \kappa^{2} \mu_{\mathrm{eff}}}{\lambda}\right)-\frac{\lambda \mu}{2 \mu_{\mathrm{eff}}} \lambda v^{2},
\end{aligned}
$$

and in the bases $\left(A_{\mathrm{NSM}}, \operatorname{Im}[S]\right)$, those for CP-odd Higgs fields are given by

$$
\begin{aligned}
\mathcal{M}_{P, 11}^{2} & =\frac{2 \mu_{\mathrm{eff}}\left(\lambda A_{\lambda}+\kappa \mu_{\mathrm{eff}}\right)}{\lambda \sin 2 \beta}, \\
\mathcal{M}_{P, 22}^{2} & =\frac{\left(\lambda A_{\lambda}+4 \kappa \mu_{\mathrm{eff}}\right) \sin 2 \beta}{4 \mu_{\mathrm{eff}}} \lambda v^{2}-\frac{3 \mu_{\mathrm{eff}}}{\lambda} \kappa A_{\kappa}-\frac{\lambda \mu}{2 \mu_{\mathrm{eff}}} \lambda v^{2}, \\
\mathcal{M}_{P, 12}^{2} & =\frac{v}{\sqrt{2}}\left(\lambda A_{\lambda}-2 \kappa \mu_{\mathrm{eff}}\right) .
\end{aligned}
$$

Three CP-even Higgs mass eigenstates $h_{i}=\left\{h, H, h_{\mathrm{s}}\right\}$ and two CP-odd Higgs mass eigenstates $a_{i}=\left\{A_{H}, A_{\mathrm{s}}\right\}$ are then obtained by

$$
\begin{aligned}
h_{i} & =V_{h_{i}}^{\mathrm{NSM}} H_{\mathrm{NSM}}+V_{h_{i}}^{\mathrm{SM}} H_{\mathrm{SM}}+V_{h_{i}}^{\mathrm{S}} \operatorname{Re}[S], \\
a_{i} & =U_{a_{i}}^{\mathrm{NSM}} A_{\mathrm{NSM}}+U_{a_{i}}^{\mathrm{S}} \operatorname{Im}[S],
\end{aligned}
$$

where $V$ and $U$ are unitary matrices used to diagonalize $\mathcal{M}_{S}^{2}$ and $\mathcal{M}_{P}^{2}$, respectively. The model also predicts a pair of charged Higgs bosons, $H^{ \pm}=\cos \beta H_{u}^{ \pm}+\sin \beta H_{d}^{ \pm}$, and their masses take the following simple form:

$$
m_{H_{ \pm}}^{2}=\frac{2 \mu_{\mathrm{eff}}}{\sin 2 \beta}\left(\frac{\kappa}{\lambda} \mu_{\mathrm{eff}}+A_{\lambda}\right)+m_{W}^{2}-\lambda^{2} v^{2} .
$$

In this study, the following properties of the Higgs bosons are utilized:

- $h$ corresponds to the scalar discovered at the LHC. The LHC Higgs data have restricted $\sqrt{\left(V_{h}^{\mathrm{NSM}}\right)^{2}+\left(V_{h}^{\mathrm{S}}\right)^{2}} \lesssim 0.1$ and $\left|V_{h}^{\mathrm{SM}}\right| \sim 1$. Furthermore, from theoretical points of view, its mass may be significantly affected by the interaction $\lambda \hat{s} \hat{H}_{u} \cdot \hat{H}_{d}$, the singlet-doublet Higgs mixing as well as the radiative correction from top/stop loops [34-36].

- $H$ and $A_{H}$ are $H_{\mathrm{NSM}^{-}}$and $A_{\mathrm{NSM}^{-}}$-dominated states. They are approximately degenerate in mass with the charged Higgs bosons if they are significantly heavier than $v$. The LHC search for extra Higgs bosons, the measured property of $h$, and the indirect constraints from $B$-physics prefer $m_{H}, m_{A_{H}} \gtrsim 200 \mathrm{GeV}$ [37] when the alignment condition is satisfied [38, 39]. It is worth noting that, although $H$ of several hundreds of $\mathrm{GeV}$ may play a significant role in the DM-nucleon scattering discussed below in specific situations [40], its contribution to the scattering is usually far below current DD experimental sensitivities. ${ }^{2}$ Thus, only the case where $H$ and $A_{H}$ are

\footnotetext{
${ }^{2}$ The $H$-mediated contribution to the scattering is proportional to $\tan ^{2} \beta / m_{H}^{4}$, and its typical size is $10^{-49} \mathrm{~cm}^{2}$ for $\tan \beta \leq 5$ (see, e.g., figure 6 in [5]). With the increase of $\tan \beta$, the lower bound of $m_{H}$ increases rapidly to satisfy the constraints from the LHC search for extra Higgs bosons (see, e.g., figure 2 in [41] for the MSSM results.). Therefore, the contribution is difficult to reach $10^{-47} \mathrm{~cm}^{2}$.
} 
above $1 \mathrm{TeV}$ is considered in this paper to simplify the analysis of DM phenomenology. This case is a theoretical hypothesis, and it can be realized by simply setting $A_{\lambda}$ a significant value, e.g., $A_{\lambda}=2 \mathrm{TeV}$ in the following numerical study. It does not change essentially the conclusions of this paper.

- $h_{s}$ and $A_{s}$ are mainly composed of the singlet field. They may be moderately light (e.g., several tens of $\mathrm{GeV}$ ) without conflicting with any collider constraints. As will be shown below, they play important roles in DM physics.

- In the case of massive charged Higgs bosons, the following approximations hold [5]:

$$
\begin{aligned}
& m_{h_{s}}^{2} \simeq \mathcal{M}_{S, 33}^{2}-\frac{\mathcal{M}_{S, 13}^{4}}{\mathcal{M}_{S, 11}^{2}-\mathcal{M}_{S, 33}^{2}}, \quad m_{A_{s}}^{2} \simeq \mathcal{M}_{P, 22}^{2}-\frac{\mathcal{M}_{P, 12}^{4}}{\mathcal{M}_{P, 11}^{2}-\mathcal{M}_{P, 22}^{2}}, \\
& \frac{V_{h}^{\mathrm{S}}}{V_{h}^{\mathrm{SM}}} \simeq \frac{\mathcal{M}_{S, 23}^{2}}{m_{h}^{2}-\mathcal{M}_{S, 33}^{2}}, \quad V_{h}^{\mathrm{NSM}} \sim 0, \quad V_{h}^{\mathrm{SM}} \simeq \sqrt{1+\left(\frac{V_{h}^{\mathrm{S}}}{V_{h}^{\mathrm{SM}}}\right)^{2}} \sim 1, \\
& \frac{V_{h_{s}}^{\mathrm{SM}}}{V_{h_{s}}^{\mathrm{S}}} \simeq \frac{\mathcal{M}_{S, 23}^{2}}{m_{h_{s}}^{2}-m_{h}^{2}}, \quad V_{h_{s}}^{\mathrm{NSM}} \sim 0, \quad V_{h_{s}}^{\mathrm{S}} \simeq \sqrt{1+\left(\frac{V_{h_{s}}^{\mathrm{SM}}}{V_{h_{s}}^{\mathrm{S}}}\right)^{2}} \sim 1, \quad U_{A_{s}}^{\mathrm{S}} \simeq 1 .
\end{aligned}
$$

It is emphasized that the mass matrices $\mathcal{M}_{S}^{2}$ and $\mathcal{M}_{P}^{2}$ differ from their corresponding ones in the $Z_{3}$-NMSSM by additional $\mu$-induced contributions. These contributions are sometimes vital in this study, e.g., a positively considerable $\mu$ can significantly reduce the mass of $h_{s}$ and $A_{s}$ so that they may act as the annihilation product of moderately light DM.

The neutralino sector comprises a Bino field $\tilde{B}^{0}$, a Wino field $\tilde{W}^{0}$, Higgsino fields $\tilde{H}_{d}^{0}$ and $\tilde{H}_{u}^{0}$, and a Singlino field $\tilde{S}^{0}$. In the bases $\psi^{0}=\left(-i \tilde{B}^{0},-i \tilde{W}^{0}, \tilde{H}_{d}^{0}, \tilde{H}_{u}^{0}, \tilde{S}^{0}\right)$, the symmetric neutralino mass matrix is given by [12]

$$
\mathcal{M}=\left(\begin{array}{ccccc}
M_{1} & 0 & -m_{Z} \sin \theta_{W} \cos \beta & m_{Z} \sin \theta_{W} \sin \beta & 0 \\
& M_{2} & m_{Z} \cos \theta_{W} \cos \beta & -m_{Z} \cos \theta_{W} \sin \beta & 0 \\
& & 0 & -\mu_{\mathrm{tot}} & -\frac{1}{\sqrt{2}} \lambda v \sin \beta \\
& & & 0 & -\frac{1}{\sqrt{2}} \lambda v \cos \beta \\
& & & & \frac{2 \kappa}{\lambda} \mu_{\mathrm{eff}}
\end{array}\right),
$$

where $M_{1}$ and $M_{2}$ are gaugino soft breaking masses and $\mu_{\mathrm{tot}} \equiv \mu_{\mathrm{eff}}+\mu$. It can be diagonalized by a rotation matrix $N$, and, subsequently, the mass eigenstates labeled in mass-ascending order are

$$
\tilde{\chi}_{i}^{0}=N_{i 1} \psi_{1}^{0}+N_{i 2} \psi_{2}^{0}+N_{i 3} \psi_{3}^{0}+N_{i 4} \psi_{4}^{0}+N_{i 5} \psi_{5}^{0}
$$

Evidently, $N_{i 3}$ and $N_{i 4}$ characterize the $\tilde{H}_{d}^{0}$ and $\tilde{H}_{u}^{0}$ components in $\tilde{\chi}_{i}^{0}$, and $N_{i 5}$ denotes the Singlino component. We add that the Higgsino mass and Singlino mass in the GNMSSM are determined by $\mu_{\text {tot }}$ and $\mu_{\text {eff }}$, respectively. As a result, $|2 \kappa / \lambda|$ may be much larger than 1 in obtaining Singlino-dominated DM, which is the starting point of this work. In addition, there is one more input parameter to describe the Singlino-Higgsino mixing system than 
in the case of the $Z_{3}$-NMSSM. These characteristics distinguish the GNMSSM from the $Z_{3}$-NMSSM, which, as introduced previously, has important implications in DM physics.

In the case of very massive gauginos and $\left|m_{\tilde{\chi}_{1}^{0}}^{2}-\mu_{\mathrm{tot}}^{2}\right| \gg \lambda^{2} v^{2}$, the following approximations for the Singlino-dominated $\tilde{\chi}_{1}^{0}[42-44]$ are obtained:

$$
\begin{aligned}
\frac{2 \kappa}{\lambda} \mu_{\mathrm{eff}} & \simeq m_{\tilde{\chi}_{1}^{0}}-\frac{1}{2} \frac{\lambda^{2} v^{2}\left(m_{\tilde{\chi}_{1}^{0}}-\mu_{\mathrm{tot}} \sin 2 \beta\right)}{m_{\tilde{\chi}_{1}^{0}}^{2}-\mu_{\mathrm{tot}}^{2}}, \quad N_{11} \sim 0, \quad N_{12} \sim 0 \\
\frac{N_{13}}{N_{15}} & =\frac{\lambda v}{\sqrt{2} \mu_{\mathrm{tot}}} \frac{\left(m_{\tilde{\chi}_{1}^{0}} / \mu_{\mathrm{tot}}\right) \sin \beta-\cos \beta}{1-\left(m_{\tilde{\chi}_{1}^{0}} / \mu_{\mathrm{tot}}\right)^{2}}, \quad \frac{N_{14}}{N_{15}}=\frac{\lambda v}{\sqrt{2} \mu_{\mathrm{tot}}} \frac{\left(m_{\tilde{\chi}_{1}^{0}} / \mu_{\mathrm{tot}}\right) \cos \beta-\sin \beta}{1-\left(m_{\tilde{\chi}_{1}^{0}} / \mu_{\mathrm{tot}}\right)^{2}}, \\
N_{15}^{2} & \simeq\left(1+\frac{N_{13}^{2}}{N_{15}^{2}}+\frac{N_{14}^{2}}{N_{15}^{2}}\right)^{-1} \\
& =\frac{\left[1-\left(m_{\tilde{\chi}_{1}^{0}} / \mu_{\mathrm{tot}}\right)^{2}\right]^{2}}{\left[\left(m_{\tilde{\chi}_{1}^{0}} / \mu_{\mathrm{tot}}\right)^{2}-2\left(m_{\tilde{\chi}_{1}^{0}} / \mu_{\mathrm{tot}}\right) \sin 2 \beta+1\right]\left(\frac{\lambda v}{\sqrt{2} \mu_{\mathrm{tot}}}\right)^{2}+\left[1-\left(m_{\tilde{\chi}_{1}^{0}} / \mu_{\mathrm{tot}}\right)^{2}\right]^{2}} .
\end{aligned}
$$

These approximations imply the relation

$$
\frac{Z_{h}}{Z_{s}}=\left(\frac{\lambda v}{\sqrt{2} \mu_{\mathrm{tot}}}\right)^{2} \frac{\left(m_{\tilde{\chi}_{1}^{0}} / \mu_{\mathrm{tot}}\right)^{2}-2\left(m_{\tilde{\chi}_{1}^{0}} / \mu_{\mathrm{tot}}\right) \sin 2 \beta+1}{\left[1-\left(m_{\tilde{\chi}_{1}^{0}} / \mu_{\mathrm{tot}}\right)^{2}\right]^{2}}
$$

where $Z_{h} \equiv N_{13}^{2}+N_{14}^{2}$ and $Z_{s} \equiv N_{15}^{2}$ represent the Higgsino and Singlino fractions in $\tilde{\chi}_{1}^{0}$, respectively, and $Z_{s}>0.5$ for the Singlino-dominated $\tilde{\chi}_{1}^{0}$. They also show that, for fixed $\tan \beta$, the Higgsino compositions in $\tilde{\chi}_{1}^{0}$ depend only on $\lambda, \mu_{\text {tot }}$, and $m_{\tilde{\chi}_{1}^{0}}$. In studying the $\tilde{\chi}_{1}^{0}$ 's properties, it is usually convenient to take the three parameters and $\kappa$ as theoretical inputs.

In this work, the following interactions are crucial:

$$
\begin{aligned}
& C_{\tilde{\chi}_{1}^{0} \tilde{\chi}_{1}^{0} h_{i}}=V_{h_{i}}^{\mathrm{SM}} C_{\tilde{\chi}_{1}^{0} \tilde{\chi}_{1}^{0} H_{\mathrm{SM}}}+V_{h_{i}}^{\mathrm{NSM}} C_{\tilde{\chi}_{1}^{0} \tilde{\chi}_{1}^{0} H_{\mathrm{NSM}}}+V_{h_{i}}^{\mathrm{S}} C_{\tilde{\chi}_{1}^{0} \tilde{\chi}_{1}^{0} R e[S]} \\
& \simeq \sqrt{2} \lambda\left[V_{h_{i}}^{\mathrm{SM}} N_{15}\left(N_{13} \sin \beta+N_{14} \cos \beta\right)+V_{h_{i}}^{\mathrm{NSM}} N_{15}\left(N_{13} \cos \beta-N_{14} \sin \beta\right)\right] \\
& +\sqrt{2} V_{h_{i}}^{\mathrm{S}}\left(\lambda N_{13} N_{14}-\kappa N_{15}^{2}\right), \\
& C_{\tilde{\chi}_{1}^{0} \tilde{\chi}_{1}^{0} a_{i}}=U_{a_{i}}^{\mathrm{NSM}} C_{\tilde{\chi}_{1}^{0} \tilde{\chi}_{1}^{0} A_{\mathrm{NSM}}}+U_{a_{i}}^{\mathrm{S}} C_{\tilde{\chi}_{1}^{0} \tilde{\chi}_{1}^{0} \operatorname{Im}[S]} \\
& \simeq-\sqrt{2} U_{a_{i}}^{\mathrm{NSM}} \lambda N_{15}\left(N_{13} \cos \beta+N_{14} \sin \beta\right)+\sqrt{2} U_{a_{i}}^{\mathrm{S}}\left(\lambda N_{13} N_{14}-\kappa N_{15}^{2}\right), \\
& C_{\tilde{\chi}_{1}^{0} \tilde{\chi}_{1}^{0} Z}=\frac{m_{Z}}{v}\left(N_{13}^{2}-N_{14}^{2}\right), \quad C_{\tilde{\chi}_{1}^{0} \tilde{\chi}_{1}^{0} G^{0}} \simeq-\sqrt{2} \lambda N_{15}\left(N_{13} \sin \beta-N_{14} \cos \beta\right), \\
& C_{h_{i} A_{s} A_{s}} \simeq \lambda v V_{h_{i}}^{\mathrm{SM}}(\lambda+\kappa \sin 2 \beta)+\lambda \kappa v V_{h_{i}}^{\mathrm{NSM}} \cos 2 \beta+\sqrt{2} V_{h_{i}}^{S}\left(\sqrt{2} \kappa^{2} v_{s}-\kappa A_{\kappa}\right) \text {. }
\end{aligned}
$$

These expressions indicate that

$$
\begin{aligned}
& C_{\tilde{\chi}_{1}^{0} \tilde{\chi}_{1}^{0} h_{s}} \sim-\sqrt{2} \kappa, \\
& C_{\tilde{\chi}_{1}^{0} \tilde{\chi}_{1}^{0} A_{s}} \sim-\sqrt{2} \kappa, \\
& C_{h_{s} A_{s} A_{s}} \sim \sqrt{2}\left(\sqrt{2} \kappa^{2} v_{s}-\kappa A_{\kappa}\right), \\
& C_{h A_{s} A_{s}} \sim \lambda v(\lambda+\kappa \sin 2 \beta), \\
& C_{H A_{s} A_{s}} \sim \lambda \kappa v, \\
& \left|C_{h_{s} A_{s} A_{s}}\right| \gg\left|C_{h A_{s} A_{s}}\right|,\left|C_{H A_{s} A_{s}}\right| \text {. }
\end{aligned}
$$

and the other interactions are suppressed. 


\subsection{DM relic density}

In the GNMSSM, Singlino-dominated DM could achieve the measured abundance by the processes $\tilde{\chi}_{1}^{0} \tilde{\chi}_{1}^{0} \rightarrow h_{s} A_{s}, t \bar{t}$, or its co-annihilation with one or more slightly heavier states, such as the next-to-lightest neutralino $\tilde{\chi}_{2}^{0}$, the lightest chargino $\tilde{\chi}_{1}^{ \pm}$, or sleptons. Under a non-relativistic approximation, the thermally averaged cross-section of DM pair annihilation can be expanded as $[3,45]$

$$
\left\langle\sigma_{A} v\right\rangle=a+b\left\langle v^{2}\right\rangle+\mathcal{O}\left(\left\langle v^{4}\right\rangle\right) \approx a+6 \frac{b}{x}
$$

where $a$ and $b$ correspond to the s- and p-wave contributions of the process, respectively, and $x \equiv m_{\tilde{\chi}_{1}^{0}} / T$ with $T$ denoting the temperature in the early Universe. After integrating the density function from $x_{F}=m / T_{F}$ at freeze-out temperature to infinity, the present relic density can be expressed as [5]

$$
\Omega h^{2}=0.12\left(\frac{80}{g_{*}}\right)^{1 / 2}\left(\frac{x_{F}}{25}\right)\left(\frac{2.3 \times 10^{-26} \mathrm{~cm}^{3} / \mathrm{s}}{\langle\sigma v\rangle_{x_{F}}}\right),
$$

where $g_{*} \sim 80$ is the number of effectively relativistic degrees of freedom, and $x_{F} \sim 25$ is obtained by solving the freeze-out equation in [45].

The key features of these annihilations are shown in the following.

1. $\tilde{\chi}_{1}^{0} \tilde{\chi}_{1}^{0} \rightarrow h_{s} A_{s}$

This process proceeds through the $s$-channel exchange of $Z$ and CP-odd Higgs bosons, and the $t$-channel exchange of neutralinos. Consequently, $\langle\sigma v\rangle_{x_{F}}^{h_{s} A_{s}}$ is given by $[5,45]$

$$
\langle\sigma v\rangle_{x_{F}}^{h_{s} A_{s}} \simeq \frac{1}{64 \pi m_{\tilde{\chi}_{1}^{0}}^{2}}\left\{\left[1-\frac{\left(m_{h_{s}}+m_{A_{s}}\right)^{2}}{4 m_{\tilde{\chi}_{1}^{0}}^{2}}\right]\left[1-\frac{\left(m_{h_{s}}-m_{A_{s}}\right)^{2}}{4 m_{\tilde{\chi}_{1}^{0}}^{2}}\right]\right\}^{1 / 2}\left|\mathcal{A}_{s}+\mathcal{A}_{t}\right|^{2},
$$

where the $s$ - and $t$-channel contributions are approximated by

$$
\begin{aligned}
& \mathcal{A}_{s} \simeq \frac{-2 m_{\tilde{\chi}_{1}^{0}} C_{\tilde{\chi}_{1}^{0} \tilde{\chi}_{1}^{0} A_{s}} C_{h_{s} A_{s} A_{s}}}{m_{A_{s}}^{2}-4 m_{\tilde{\chi}_{1}^{0}}^{2}}, \\
& \mathcal{A}_{t} \simeq-2 C_{\tilde{\chi}_{1}^{0} \tilde{\chi}_{1}^{0} h_{s}} C_{\tilde{\chi}_{1}^{0} \tilde{\chi}_{1}^{0} A_{s}}\left[1+\frac{2 m_{A_{s}}^{2}}{4 m_{\tilde{\chi}_{1}^{0}}^{2}-\left(m_{h_{s}}^{2}+m_{A_{s}}^{2}\right)}\right],
\end{aligned}
$$

respectively, if there are no resonant contributions. With the formulae from eqs. (2.12)-(2.16), one can learn that, once the involved particles' masses are fixed, the density is mainly determined by the parameter $\kappa$. The authors of ref. [5] estimated the value of $\kappa$ to predict the density, and concluded that

$$
|\kappa| \sim 0.15 \times\left(\frac{m_{\tilde{\chi}_{1}^{0}}}{300 \mathrm{GeV}}\right)^{1 / 2}
$$

in the case of $\left|\mathcal{A}_{t}\right| \gg\left|\mathcal{A}_{s}\right|$. 


\section{2. $\tilde{\chi}_{1}^{0} \tilde{\chi}_{1}^{0} \rightarrow t \bar{t}$}

This annihilation is mediated by the s-channel exchange of $Z$ and Higgs bosons. Owing to the largeness of top quark mass, its amplitude is mainly contributed by the longitudinal polarization of the $Z$ boson when the mediators of the Higgs bosons are far off-shell. As a result, $\langle\sigma v\rangle_{x_{F}}^{t \bar{t}}$ is approximated by [5]

$$
\langle\sigma v\rangle_{x_{F}}^{t \bar{t}} \sim 2 \times 10^{-26} \frac{\mathrm{cm}^{3}}{\mathrm{~s}}\left(\frac{\left|C_{\tilde{\chi}_{1}^{0} \tilde{\chi}_{1}^{0} G^{0}}\right|}{0.1}\right)^{2}\left(\frac{m_{\tilde{\chi}_{1}^{0}}}{300 \mathrm{GeV}}\right)^{-2}
$$

where

$$
C_{\tilde{\chi}_{1}^{0} \tilde{\chi}_{1}^{0} G^{0}} \simeq-\sqrt{2} \lambda N_{15}\left(N_{13} s_{\beta}-N_{14} c_{\beta}\right) \simeq \frac{\lambda^{2} v}{\mu_{\text {tot }}} \frac{Z_{s}\left(m_{\tilde{\chi}_{1}^{0}} / \mu_{\text {tot }}\right) \cos 2 \beta}{1-\left(m_{\tilde{\chi}_{1}^{0}} / \mu_{\text {tot }}\right)^{2}} .
$$

These formulae reflect that a moderately large $\lambda$ is needed to account for the density. As will be shown below, this situation usually leads to sizable DM-nucleon scattering rates.

3. Co-annihilation with Higgsino-dominated electroweakinos

This annihilation affects the abundance when the mass splitting between $\tilde{\chi}_{1}^{0}$ and the Higgsino-dominated electroweakinos is less than approximately $10 \%[45,46]$. In this case, $\sigma$ in eq. (2.18) should be replaced by $\sigma_{\text {eff }}$ given by

$$
\sigma_{\mathrm{eff}}=\sum_{a, b}^{N} \sigma_{a b} \frac{g_{a} g_{b}}{g_{\mathrm{eff}}^{2}}\left(1+\Delta_{a}\right)^{3 / 2}\left(1+\Delta_{b}\right)^{3 / 2} \times \exp \left[-x\left(\Delta_{a}+\Delta_{b}\right)\right] .
$$

In this formula, $\sigma_{a b}$ is the cross-section of the annihilation $a b \rightarrow X Y$ with $a, b=$ $\tilde{\chi}_{1}^{0}, \tilde{\chi}_{2}^{0}, \tilde{\chi}_{1}^{ \pm}, \cdots$ and $X, Y$ denoting any possible final states, $\Delta_{i} \equiv\left(m_{i}-m_{\tilde{\chi}_{1}^{0}}\right) / m_{\tilde{\chi}_{1}^{0}}$ $(i=a, b)$ represents the mass splitting between $\tilde{\chi}_{1}^{0}$ and the initial state $i$, and $x \equiv$ $m_{\tilde{\chi}_{1}^{0}} / T . g_{i}$ denotes the internal degrees of freedom for the initial state $i$, which is 2 for a Majorana fermion and 4 for a Dirac fermion, and $g_{\text {eff }}$ is defined by

$$
g_{\mathrm{eff}} \equiv \sum_{a}^{N} g_{a}\left(1+\Delta_{a}\right)^{3 / 2} \exp \left(-x \Delta_{a}\right) .
$$

It is evident that $\sigma_{\text {eff }}$ decreases monotonously as the co-annihilating particles depart from $\tilde{\chi}_{1}^{0}$ in mass.

Finally, the annihilations $\tilde{\chi}_{1}^{0} \tilde{\chi}_{1}^{0} \rightarrow h A_{s}, h_{s} h_{s}$ are briefly discussed. The former process proceeds in a similar way as $\tilde{\chi}_{1}^{0} \tilde{\chi}_{1}^{0} \rightarrow h_{s} A_{s}$. From the expressions of $C_{\tilde{\chi}_{1}^{0} \tilde{\chi}_{1}^{0} h}$ and $C_{h A_{s} A_{s}}$ and the fact that $C_{\tilde{\chi}_{1}^{0} \tilde{\chi}_{1}^{0} h_{s}} \gg C_{\tilde{\chi}_{1}^{0} \tilde{\chi}_{1}^{0} h}$ and $C_{h_{s} A_{s} A_{s}} \gg C_{h A_{s} A_{s}}$ in most cases, one can conclude that the abundance is significantly affected only when $\lambda$ and $V_{h}^{S}$ are tremendously large. This condition, however, has been tightly limited by the XENON-1T and LHC experiments. Process $\tilde{\chi}_{1}^{0} \tilde{\chi}_{1}^{0} \rightarrow h_{s} h_{s}$ proceeds by the s-channel exchange of the CP-even Higgs bosons and the t-channel exchange of the neutralinos. It is a p-wave process [47], so its effect on the abundance becomes crucial only when $\kappa$ is large and $\tilde{\chi}_{1}^{0} \tilde{\chi}_{1}^{0} \rightarrow h_{s} A_{s}$ is phase-spacesuppressed. This situation happens only in some corners of the theory's parameter space. 


\subsection{DM-nucleon scattering cross-sections}

In the limit of very massive squarks, the SD scattering of $\tilde{\chi}_{1}^{0}$ with nucleons is mediated by a $Z$ boson. The scattering cross-section is then given by [43, 44]

$$
\sigma_{\tilde{\chi}_{1}^{0}-N}^{\mathrm{SD}} \simeq C_{N} \times\left(\frac{N_{13}^{2}-N_{14}^{2}}{0.1}\right)^{2}
$$

where $C_{p} \simeq 4 \times 10^{-4} \mathrm{pb}$ for protons and $C_{n} \simeq 3.1 \times 10^{-4} \mathrm{pb}$ for neutrons, and

$$
N_{13}^{2}-N_{14}^{2}=\left(\frac{\lambda v}{\sqrt{2} \mu_{\mathrm{tot}}}\right)^{2} \frac{Z_{s} \cos 2 \beta}{1-\left(m_{\tilde{\chi}_{1}^{0}} / \mu_{\mathrm{tot}}\right)^{2}},
$$

from the formulae in eq. (2.10). These expressions indicate that the SD cross-section is proportional to $\left(\lambda v / \mu_{\text {tot }}\right)^{4}$ and it increases as $m_{\tilde{\chi}_{1}^{0}}$ approaches from below to $\mu_{\text {tot }}$.

The SI scattering is mainly contributed by the $t$-channel exchange of the CP-even Higgs bosons. Correspondingly, the cross-section is given by [48, 49]

$$
\sigma_{\tilde{\chi}_{1}^{0}-N}^{S I}=\frac{4 \mu_{r}^{2}}{\pi}\left|f^{(N)}\right|^{2},
$$

where $N=p, n$ denotes proton and neutron, respectively, and $\mu_{r}$ is the reduced mass of $\tilde{\chi}_{1}^{0}$ and nucleon given by $\mu_{r} \equiv m_{N} m_{\tilde{\chi}_{1}^{0}} /\left(m_{N}+m_{\tilde{\chi}_{1}^{0}}\right)$. The effective couplings $f^{(N)}$ are

$$
f^{(N)}=\sum_{h_{i}=\left\{h, H, h_{\mathrm{s}}\right\}} f_{h_{i}}^{(N)}=\sum_{h_{i}=\left\{h, H, h_{\mathrm{s}}\right\}} \frac{C_{\tilde{\chi}_{1}^{0} \tilde{\chi}_{1}^{0} h_{i}} C_{N N h_{i}}}{2 m_{h_{i}}^{2}},
$$

where $C_{N N h_{i}}$ represents the $h_{i}$-nucleon coupling, which is given by

$$
C_{N N h_{i}}=-\frac{m_{N}}{v}\left[F_{d}^{(N)}\left(V_{h_{i}}^{\mathrm{SM}}-\tan \beta V_{h_{i}}^{\mathrm{NSM}}\right)+F_{u}^{(N)}\left(V_{h_{i}}^{\mathrm{SM}}+\frac{1}{\tan \beta} V_{h_{i}}^{\mathrm{NSM}}\right)\right],
$$

with $F_{d}^{(N)} \equiv f_{d}^{(N)}+f_{s}^{(N)}+\frac{2}{27} f_{G}^{(N)}$ and $F_{u}^{(N)} \equiv f_{u}^{(N)}+\frac{4}{27} f_{G}^{(N)}$. The nucleon form factors $f_{q}^{(N)}=m_{N}^{-1}\left\langle N\left|m_{q} q \bar{q}\right| N\right\rangle$ for $q=u, d, s$ represent the normalized light quark contribution to nucleon mass, and $f_{G}^{(N)}=1-\sum_{q=u, d, s} f_{q}^{(N)}$ denotes other heavy quarks' mass fraction in the nucleon [50, 51]. For the default settings of the package micrOMEGAs about $f_{q}^{N}[52-54]$, $F_{u}^{p} \simeq F_{u}^{n} \simeq 0.15$ and $F_{d}^{p} \simeq F_{d}^{n} \simeq 0.13$. Consequently, one can conclude that $\sigma_{\tilde{\chi}_{1}^{0}-p}^{S I} \simeq \sigma_{\tilde{\chi}_{1}^{0}-n}^{S I}$.

In the case of very massive charged Higgs bosons, $V_{h}^{\mathrm{NSM}} \sim 0, V_{h_{s}}^{\mathrm{NSM}} \sim 0$, and the $H$-mediated contribution can be safely neglected since it is further suppressed by a factor $1 / m_{H}^{4}$. Consequently, the SI cross-section is given by [14]

$$
\sigma_{\tilde{\chi}_{1}^{0}-N}^{\mathrm{SI}} \simeq 5 \times 10^{-45} \mathrm{~cm}^{2}\left(\frac{\mathcal{A}}{0.1}\right)^{2},
$$

where

$$
\mathcal{A}=\left(\frac{125 \mathrm{GeV}}{m_{h}}\right)^{2} V_{h}^{\mathrm{SM}} C_{\tilde{\chi}_{1}^{0} \tilde{\chi}_{1}^{0} h}+\left(\frac{125 \mathrm{GeV}}{m_{h_{s}}}\right)^{2} V_{h_{s}}^{\mathrm{SM}} C_{\tilde{\chi}_{1}^{0} \tilde{\chi}_{1}^{0} h_{s}} .
$$


Furthermore, using the formulae from eqs. (2.10)-(2.15), one may conclude that

$$
\begin{aligned}
C_{\tilde{\chi}_{1}^{0} \tilde{\chi}_{1}^{0} h} \simeq & \frac{\mu_{\text {tot }}}{v}\left(\frac{\lambda v}{\mu_{\text {tot }}}\right)^{2} \frac{Z_{s} V_{h}^{\mathrm{SM}}\left(m_{\tilde{\chi}_{1}^{0}} / \mu_{\mathrm{tot}}-\sin 2 \beta\right)}{1-\left(m_{\tilde{\chi}_{1}^{0}} / \mu_{\mathrm{tot}}\right)^{2}}+\frac{\lambda}{2 \sqrt{2}}\left(\frac{\lambda v}{\mu_{\mathrm{tot}}}\right)^{2} \frac{Z_{s} V_{h}^{\mathrm{S}} \sin 2 \beta}{\left[1-\left(m_{\tilde{\chi}_{1}^{0}} / \mu_{\mathrm{tot}}\right)^{2}\right]} \\
& -\sqrt{2} \kappa Z_{s} V_{h}^{\mathrm{S}}\left[1+\left(\frac{\lambda v}{\sqrt{2} \mu_{\mathrm{tot}}}\right)^{2} \frac{1}{1-\left(m_{\tilde{\chi}_{1}^{0}} / \mu_{\mathrm{tot}}\right)^{2}} \frac{\mu_{\mathrm{eff}}}{\mu_{\mathrm{tot}}}\right], \\
C_{\tilde{\chi}_{1}^{0} \tilde{\chi}_{1}^{0} h_{s}} \simeq & \frac{\mu_{\text {tot }}}{v}\left(\frac{\lambda v}{\mu_{\mathrm{tot}}}\right)^{2} \frac{Z_{s} V_{h_{s}}^{\mathrm{SM}}\left(m_{\tilde{\chi}_{1}^{0}} / \mu_{\mathrm{tot}}-\sin 2 \beta\right)}{1-\left(m_{\tilde{\chi}_{1}^{0}} / \mu_{\mathrm{tot}}\right)^{2}}+\frac{\lambda}{2 \sqrt{2}}\left(\frac{\lambda v}{\mu_{\mathrm{tot}}}\right)^{2} \frac{Z_{s} V_{h_{s}}^{\mathrm{S}} \sin 2 \beta}{\left[1-\left(m_{\tilde{\chi}_{1}^{0}} / \mu_{\mathrm{tot}}\right)^{2}\right]} \\
& -\sqrt{2} \kappa Z_{s} V_{h_{s}}^{\mathrm{S}}\left[1+\left(\frac{\lambda v}{\sqrt{2} \mu_{\mathrm{tot}}}\right)^{2} \frac{1}{1-\left(m_{\tilde{\chi}_{1}^{0}} / \mu_{\mathrm{tot}}\right)^{2}} \frac{\mu_{\mathrm{eff}}}{\mu_{\mathrm{tot}}}\right] .
\end{aligned}
$$

These formulae indicate that, if the LHC-discovered scalar is pure SM-like, i.e., $V_{h}^{\mathrm{SM}}=1$, $V_{h}^{\mathrm{S}}=0$, and $V_{h_{s}}^{\mathrm{SM}}=0$, the expression of $\mathcal{A}$ is simplified as

$$
\mathcal{A}=\left(\frac{125 \mathrm{GeV}}{m_{h}}\right)^{2} \frac{\mu_{\mathrm{tot}}}{v}\left(\frac{\lambda v}{\mu_{\mathrm{tot}}}\right)^{2} \frac{Z_{s}\left(m_{\tilde{\chi}_{1}^{0}} / \mu_{\mathrm{tot}}-\sin 2 \beta\right)}{1-\left(m_{\tilde{\chi}_{1}^{0}} / \mu_{\mathrm{tot}}\right)^{2}} .
$$

To suppress the SI cross-section, either $\lambda$ is small or $m_{\tilde{\chi}_{1}^{0}} / \mu_{\text {tot }} \simeq \sin 2 \beta$, which is called the blind-spot condition of the NMSSM [43, 48]. However, if $h_{s}$ contains a sizable SM Higgs field component, e.g., $V_{h_{s}}^{\mathrm{SM}}>0.1$, the $h_{s}$-mediated contribution may be crucial when $h_{s}$ is much lighter than $h$. This may drastically cancel the $h$-mediated contribution. In either case, the SI cross-section is usually dominated by the $h$-mediated contribution, and it is sensitive to the parameters $\lambda$ and $\mu_{\text {tot }}$. Note that this feature still holds when the $H$-mediated contribution is not negligibly small. Therefore, the DM direct detection experiments can tightly limit these two parameters. Finally, it is noticeable that $\kappa$ may also affect the cross-section significantly, but it occurs only when $\kappa \gg \lambda$ and $V_{h}^{\mathrm{S}}$ is sizable. This situation is different from that of the SD cross-section, which is insensitive to $\kappa$ in all cases.

\section{Numerical results}

In this section, the characteristics of the Singlino-dominated DM are demonstrated by numerical results. A likelihood function is constructed for the nest sampling algorithm adopted in this work to guide sophisticated scans over the $\mu$ NMSSM's parameter space [56, 57]. The scanned samples are studied by statistical quantities such as the posterior probability density function (PDF) in Bayesian statistics and the profile likelihood (PL) in frequency statistics. The former reflects the preference of the obtained samples to the input parameters, and the latter, however, represents the theory's capability to explain experimental data. These statistical quantities have been introduced concisely in [58] and are applied to scrutinize the phenomenology of the NMSSM's seesaw extensions $[10,59,60]$. 


\begin{tabular}{|c|c|c|c|c|c|}
\hline Parameter & Value & Parameter & Value & Parameter & Value \\
\hline$A_{\lambda}$ & $2 \mathrm{TeV}$ & $m_{\tilde{q}}$ & $2 \mathrm{TeV}$ & $m_{\tilde{l}}$ & $2 \mathrm{TeV}$ \\
$M_{1}$ & $3 \mathrm{TeV}$ & $M_{2}$ & $3 \mathrm{TeV}$ & $M_{3}$ & $3 \mathrm{TeV}$ \\
\hline
\end{tabular}

Table 1. Setting of different fields' soft-breaking parameters. $A_{\lambda}, m_{\tilde{q}}, m_{\tilde{l}}$, and $M_{i}(\mathrm{i}=1,2,3)$ are for Higgs, squarks, sleptons, and different gaugino fields, respectively.

\subsection{Research strategy}

The following likelihood function is taken:

$$
\mathcal{L}=\mathcal{L}_{\Omega h^{2}} \times \mathcal{L}_{D D} \times \mathcal{L}_{I D D} \times \mathcal{L}_{\mathrm{Higgs}} \times \mathcal{L}_{B}
$$

where $\mathcal{L}_{\Omega h^{2}}$ is a Gaussian likelihood function for the measured DM abundance, $\mathcal{L}_{D D}$ encodes the upper bounds on the SI and SD cross-sections from the XENON-1T experiments [6,61], and $\mathcal{L}_{I D D}$ incorporates the DM indirect search information of the Fermi-LAT experiment $[62,63] . \mathcal{L}_{\text {Higgs }}$ includes a fit of the $h$ 's property to corresponding data of the LHC by the code HiggsSignal-2.2.3 [64]. It also includes the constraints from the direct search for extra Higgs bosons at the LEP, Tevatron, and LHC, which are implemented by the code HiggsBounds-5.3.2 [65]. Finally, $\mathcal{L}_{B}$ takes a Gaussian form to describe the measured branching ratios of $B \rightarrow X_{s} \gamma$ and $B_{s}^{0} \rightarrow \mu^{+} \mu^{-}$[66]. The concrete expression of $\mathcal{L}$ was given in our previous works [59,60].

The following parameter space is scanned:

$$
\begin{aligned}
& 0<\lambda \leq 0.70, \quad|\kappa| \leq 0.70, \quad 1 \leq \tan \beta \leq 60, \quad\left|A_{t}\right| \leq 5 \mathrm{TeV} \\
& 0<\mu_{\text {eff }} \leq 500 \mathrm{GeV}, \quad 100 \mathrm{GeV} \leq\left|\mu_{\text {tot }}\right| \leq 500 \mathrm{GeV}, \quad\left|A_{\kappa}\right| \leq 1000 \mathrm{GeV},
\end{aligned}
$$

by assuming the input parameters are flat distributed. Upper bounds are imposed on $\lambda$ and $\kappa$ to keep the theory perturbative up to the Grand Unification scale. $\mu_{\mathrm{eff}}, \mu_{\mathrm{tot}}$, and $A_{\kappa}$ are restricted within relatively narrow ranges to naturally break the electroweak symmetry, and the parameter $A_{t}$ is varied to include the critical radiative correction of the top-stop loops to Higgs mass. In addition, a lower bound is set on $\left|\mu_{\text {tot }}\right|$ by the LEP search for electroweakinos [66]. For the other less crucial parameters, their values are fixed as in table 1.

The package SARAH-4.14.3 [67-70] was utilized to build the $\mu$ NMSSM's model file, and the codes SPheno-4.0.3 [71, 72] and FlavorKit [73] were used to generate the particle spectrum and calculate low-energy flavor observables, respectively. The Singlino-dominated neutralino is assumed to be the sole DM candidate in the Universe and the observables in DM physics are computed with the package MicrOMEGAs 5.0.4 [55, 74-78]. We consider $h \equiv h_{1}$ and $h \equiv h_{2}$ scenarios, where $h_{1}$ and $h_{2}$ denote the lightest and the next-tolightest Higgs bosons. Details of each obtained sample are reported for further analysis. For example, all processes that affect the abundance are listed and their fractions to the total DM annihilation cross-section at the freeze-out temperature are presented. This information helps identify the dominant contribution to the abundance. 


\begin{tabular}{|c|c|c|c|c|}
\hline & \multicolumn{2}{|c|}{$h \equiv h_{1}$ scenario } & \multicolumn{2}{c|}{$h \equiv h_{2}$ scenario } \\
\hline Parameters & $1 \sigma$ & $2 \sigma$ & $1 \sigma$ & $2 \sigma$ \\
\hline$\lambda$ & $0.04 \sim 0.24$ & $0.02 \sim 0.41$ & $0.05 \sim 0.18$ & $0.03 \sim 0.31$ \\
\hline$\kappa$ & $-0.25 \sim 0.31$ & $-0.31 \sim 0.41$ & $-0.17 \sim 0.22$ & $-0.30 \sim 0.30$ \\
\hline $\tan \beta$ & $5.9 \sim 23.3$ & $4.7 \sim 49.3$ & $5.7 \sim 12.1$ & $5.1 \sim 20.2$ \\
\hline$\mu$ & $245 \sim 420$ & $163 \sim 467$ & $150 \sim 319$ & $51 \sim 408$ \\
\hline$\mu_{\text {eff }}$ & $29 \sim 118$ & $14 \sim 253$ & $33 \sim 148$ & $18 \sim 298$ \\
\hline$\mu_{\text {tot }}$ & $325 \sim 475$ & $255 \sim 495$ & $225 \sim 425$ & $136 \sim 475$ \\
\hline$A_{\kappa}$ & $-375 \sim 128$ & $-576 \sim 397$ & $-652 \sim 339$ & $-776 \sim 534$ \\
\hline$A_{t}$ & $3148 \sim 4248$ & $2849 \sim 4448$ & $2651 \sim 4150$ & $-3845 \sim 4550$ \\
\hline$m_{\tilde{\chi}_{1}^{0}}$ & $240 \sim 407$ & $182 \sim 466$ & $181 \sim 346$ & $118 \sim 404$ \\
\hline
\end{tabular}

Table 2. One-dimensional $1 \sigma$ and $2 \sigma$ credible regions for input parameters in $h \equiv h_{1}$ and $h \equiv h_{2}$ scenarios, where $h, h_{1}$, and $h_{2}$ denote the SM-like Higgs boson and the lightest and next-to-lightest $\mathrm{CP}$-even Higgs boson, respectively. Dimensional parameters are in units of $\mathrm{GeV}$.

\begin{tabular}{|c|c|c|c|}
\hline \multicolumn{4}{|c|}{$h \equiv h_{1}$ scenario: $\ln \mathrm{Z}=-65.79 \pm 0.046$} \\
\hline$\tilde{\chi}_{1}^{0} \tilde{\chi}_{1}^{0} \rightarrow \mathrm{h}_{\mathrm{s}} \mathrm{A}_{\mathrm{s}}$ & $\tilde{\chi}_{1}^{0} \tilde{\chi}_{1}^{0} \rightarrow \mathrm{t} \overline{\mathrm{t}}$ & $\tilde{\chi}_{1}^{0} \tilde{\chi}_{1}^{0} \rightarrow \mathrm{h}_{\mathrm{s}} \mathrm{h}_{\mathrm{s}}$ & Co-annihilation \\
\hline $88 \%$ & $8 \%$ & $3 \%$ & $0.7 \%$ \\
\hline \multicolumn{4}{|c|}{$h \equiv h_{2}$ scenario: $\ln \mathrm{Z}=-68.23 \pm 0.051$} \\
\hline$\tilde{\chi}_{1}^{0} \tilde{\chi}_{1}^{0} \rightarrow \mathrm{h}_{\mathrm{s}} \mathrm{A}_{\mathrm{s}}$ & $\tilde{\chi}_{1}^{0} \tilde{\chi}_{1}^{0} \rightarrow \mathrm{t} \overline{\mathrm{t}}$ & Co-annihilation & $h$-funnel \\
\hline $76 \%$ & $12 \%$ & $11.6 \%$ & $0.3 \%$ \\
\hline
\end{tabular}

Table 3. Dominant annihilation channels and their normalized posterior probabilities for $h \equiv h_{1}$ and $h \equiv h_{2}$ scenarios. In obtaining the values in this table, each sample's most critical channel for the abundance was identified and sequentially used to classify the samples. The posterior probability densities of the same type of samples were then summed.

\subsection{Posterior probability density}

In Bayesian statistics, the one-dimensional marginal posterior PDF $P(\theta)$ with $\theta$ being an input parameter is defined by integrating the posterior PDF over the other input parameters. This decides the one-dimensional $1 \sigma(2 \sigma)$ credible region by requiring that the region's posterior probability accounts for $68 \%(95 \%)$ of the total probability [58]. In this study, each sample's posterior probability density is calculated by the nest sampling algorithm and the $1 \sigma$ and $2 \sigma$ credible regions for the input parameters are determined. The results are presented in table 2. Compared with the scanned region, one can learn that some parameters' favored range is reduced significantly. For example, $\lambda$ and $|\kappa|$ prefer the range $\lambda \lesssim 0.4$ and $|\kappa| \lesssim 0.3$ because, as mentioned above, it is more easily consistent with various DM measurements.

In table 3, dominant annihilation channels for the obtained samples and their posterior probability are listed. This table shows that $\tilde{\chi}_{1}^{0} \tilde{\chi}_{1}^{0} \rightarrow h_{s} A_{s}$ is the dominant channel for most cases, and $\tilde{\chi}_{1}^{0} \tilde{\chi}_{1}^{0} \rightarrow t \bar{t}$ is another popular channel. In contrast, the co-annihilation is vital only in rare cases in the $h \equiv h_{1}$ scenario. The characteristics of these channels 
were introduced in the preceding section. In the following, only several new features after elaborated analysis of the samples are listed.

- Any of $\tilde{\chi}_{1}^{0} \tilde{\chi}_{1}^{0} \rightarrow h_{s} A_{s}$, the co-annihilation, and the $h$-funnel can be fully responsible for the DM abundance. Compared with the first mechanism for the abundance, the latter two need a stricter condition, i.e., $\left|m_{\tilde{\chi}_{1}^{0}}\right| \simeq\left|\mu_{\text {tot }}\right|$ and $m_{\tilde{\chi}_{1}^{0}} \simeq m_{h} / 2$, respectively. Therefore, their Bayesian evidence is relatively small. ${ }^{3}$

- Owing to the tight constraints of the XENON-1T experiments on $\lambda, \tilde{\chi}_{1}^{0} \tilde{\chi}_{1}^{0} \rightarrow t \bar{t}$ contributes to the total annihilation cross-section at the freeze-out temperature by less than $50 \%$. This fact implies that, although $\tilde{\chi}_{1}^{0} \tilde{\chi}_{1}^{0} \rightarrow t \bar{t}$ is the most considerable contribution in some cases, it must be aided by other processes to achieve the correct abundance.

- Owing to its p-wave nature, $\tilde{\chi}_{1}^{0} \tilde{\chi}_{1}^{0} \rightarrow h_{s} h_{s}$ contributes to the abundance by, at most, $40 \%$. This situation is quite similar to that of $\tilde{\chi}_{1}^{0} \tilde{\chi}_{1}^{0} \rightarrow t \bar{t}$. Furthermore, since $\tilde{\chi}_{1}^{0} \tilde{\chi}_{1}^{0} \rightarrow$ $h_{s} h_{s}$ dominates over $\tilde{\chi}_{1}^{0} \tilde{\chi}_{1}^{0} \rightarrow h_{s} A_{s}$ only when the strict conditions of $m_{\tilde{\chi}_{1}^{0}}>m_{h_{s}}$, $2\left|m_{\tilde{\chi}_{1}^{0}}\right| \lesssim\left(m_{h_{s}}+m_{A_{s}}\right)$ and $|\kappa| \gtrsim 0.3$ are satisfied, its evidence tends to be small.

The next object of focus is the Bayesian evidence for different scenarios. Our calculation shows that $\ln Z=-65.79 \pm 0.046$ for the $h \equiv h_{1}$ scenario and $\ln Z=-68.21 \pm 0.051$ for the $h \equiv h_{2}$ scenario. Since Jeffreys' scale defined by $\delta \ln Z \equiv \ln \left(Z_{h_{1}} / Z_{h_{2}}\right)$ [80] is 2.42, it is concluded that the considered experiments prefer the $h \equiv h_{1}$ scenario slightly to the $h \equiv h_{2}$ scenario. $^{4}$ One reason for this conclusion is that the latter scenario needs tuning of its parameter space to explain the $125 \mathrm{GeV}$ Higgs data [34]. Also compared in the present paper is the Bayesian evidence of the $\mu \neq 0$ and $\mu=0$ cases (note that the $\mu=0$ case corresponds to the $Z_{3}$-NMSSM), and it is found that the Jeffrey's-scale result is 8.05. This result implies that the experiments strongly favor the $\mu \neq 0$ case because it is readily consistent with the DM experiments. Realizing that the evidence relies on the prior probability assumptions, it is recalculated by assuming that $\lambda$ and $\kappa$ are log-distributed and the other input parameters are flat-distributed. It is found that Jeffrey's scale changes only slightly after recalculation.

Finally, the experimentally favored parameter space of the $\mu$ NMSSM is compared with that of the $Z_{3}$-NMSSM, which was obtained in our recent work [14]. It is found that they are quite different. The reason is, as mentioned before, that the additional parameter $\mu$ contributes to the Higgs and neutralino mass matrices, and, consequently, the DM physics differs significantly.

\footnotetext{
${ }^{3}$ Bayesian evidence is usually defined by $Z(D \mid M) \equiv \int P(D \mid O(M, \Theta)) P(\Theta \mid M) \prod d \Theta_{i}$, where $P(\Theta \mid M)$ is the prior PDF of input parameters $\Theta=\left(\Theta_{1}, \Theta_{2}, \cdots\right)$ in model $M$ and $P(D \mid O(M, \Theta)) \equiv \mathcal{L}(\Theta)$ is the likelihood function for observables $O$, which considers both theoretical predictions, $O(M, \Theta)$, and experimental data, $D$. Computationally, the evidence represents an average likelihood in the surveyed parameter space. It depends on the priors of the model's parameters. For different scenarios in a theory, the larger $Z$ is, the more readily it is for the corresponding scenario to agree with the data. The details of Bayesian evidence and its applications were presented in, e.g., [79].

${ }^{4}$ Given two scenarios to be compared together, Jeffreys' scale provides a calibrated spectrum of significance for the relative strength of the scenarios [80]. For the application of the Jeffreys' scale in particle physics, see, for e.g., [81].
} 

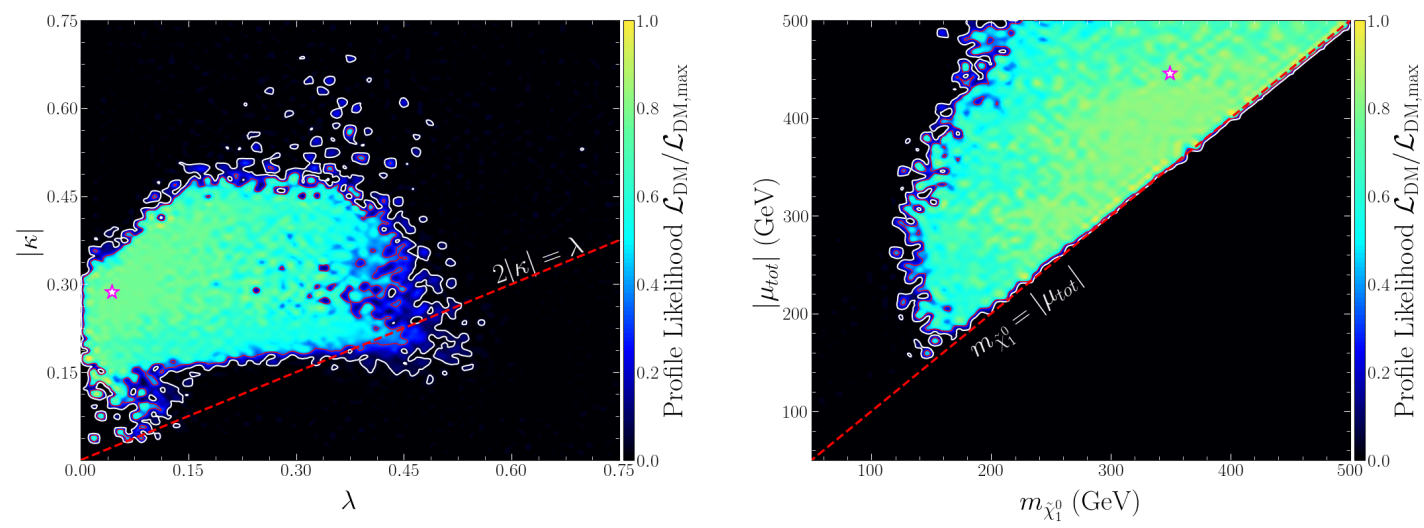

Figure 1. Two-dimensional profile likelihood of function $\mathcal{L}_{\mathrm{DM}}$ in eq. (3.3) for $h \equiv h_{1}$ scenario, which is projected onto $|\kappa|-\lambda$ and $\left|\mu_{\text {tot }}\right|-m_{\tilde{\chi}_{1}^{0}}$ planes of $\mu$ NMSSM. Since $\chi_{\text {Best }}^{2} \simeq 0$ for the best point (marked with a star symbol in figure), the boundaries for $1 \sigma$ (red solid line) and $2 \sigma$ (white solid line) confidence intervals correspond to $\chi^{2} \simeq 2.3$ and $\chi^{2} \simeq 6.18$, respectively. Dotted-dashed line represents relation $2 \kappa=\lambda$. Points around $m_{\tilde{\chi}_{1}^{0}} \simeq\left|\mu_{\text {tot }}\right|$ on right-hand panel obtained correct abundance by co-annihilation. This figure shows $\mu$ NMSSM's parameter region that can well explain DM experiments.
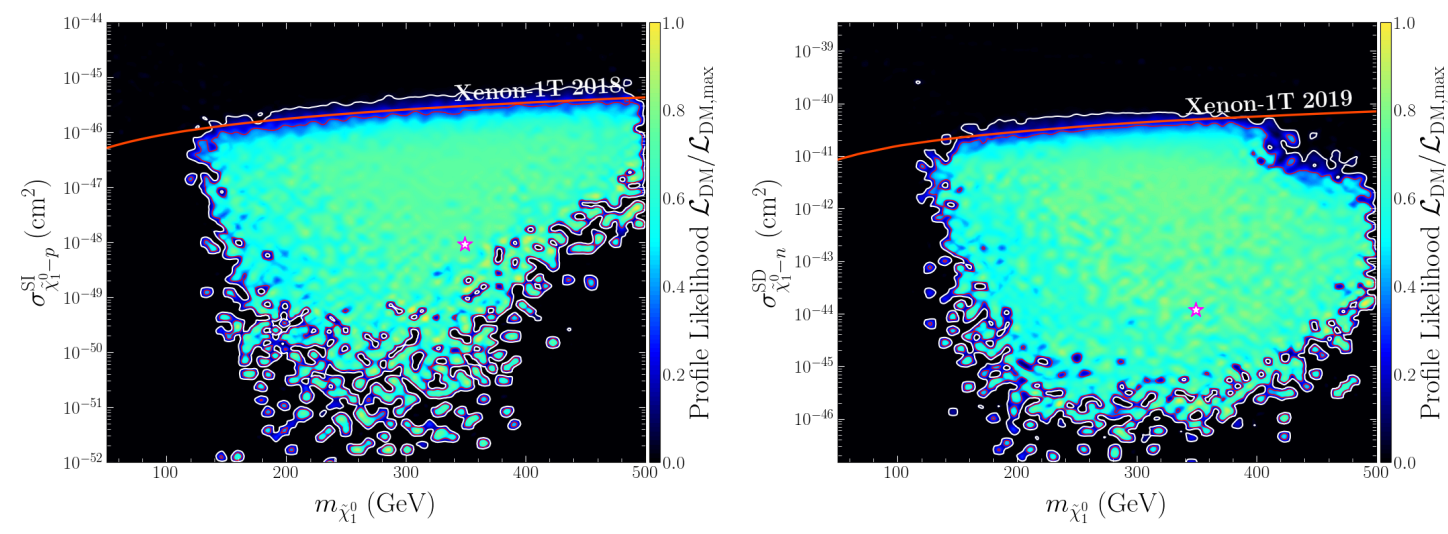

Figure 2. Same as figure 1, but for profile likelihood projected onto $\sigma_{\tilde{\chi}_{1}^{0}-p}^{\mathrm{SI}}-m_{\tilde{\chi}_{1}^{0}}$ and $\sigma_{\tilde{\chi}_{1}^{0}-n}^{\mathrm{SD}}-m_{\tilde{\chi}_{1}^{0}}$ plane. Red curve denotes latest XENON-1T's bound on SI and SD cross-section.

\subsection{Profile likelihood of DM physics}

The object of focus in this subsection is the theory's capability to explain the DM experiments. DM's two-dimensional (2D) PL is defined as

$$
\mathcal{L}_{\mathrm{DM}}\left(\Theta_{A}, \Theta_{B}\right)=\underset{\Theta_{1}, \cdots, \Theta_{A-1}, \Theta_{A+1}, \cdots, \Theta_{B-1}, \Theta_{B+1}, \cdots}{\max }\left\{\mathcal{L}_{\Omega h^{2}} \times \mathcal{L}_{D D} \times \mathcal{L}_{I D D}\right\}(\Theta),
$$

where $\Theta=\left(\Theta_{1} \equiv \lambda, \Theta_{2} \equiv \kappa, \cdots\right)$ are the input parameters of $\mathcal{L}_{i}\left(i=\Omega h^{2}, \cdots\right)$, and the maximization is realized by varying the parameters other than $\Theta_{A}$ and $\Theta_{B}$. The $1 \sigma$ $(2 \sigma)$ confidence interval on the $\Theta_{A}-\Theta_{B}$ plane is determined by the criteria of $\left(\delta \chi^{2} \equiv\right.$ $\left.\chi^{2}-\chi_{\text {Best }}^{2}\right) \leq 2.3\left(\delta \chi^{2} \leq 6.18\right)$, where $\chi^{2} \equiv-2 \ln \mathcal{L}_{\mathrm{DM}}\left(\Theta_{A}, \Theta_{B}\right)$ and $\chi_{\text {Best }}^{2}$ denotes the best point's $\chi^{2}$. Two subtleties about $\mathcal{L}_{\mathrm{DM}}\left(\Theta_{A}, \Theta_{B}\right)$ must be clarified. One is that it is 

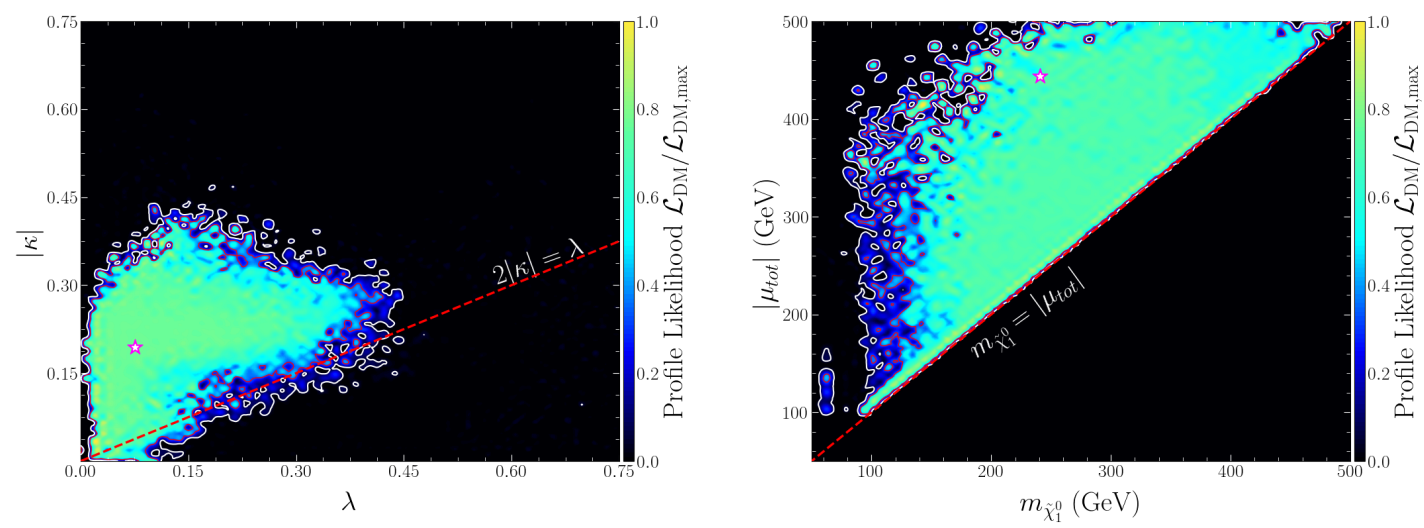

Figure 3. Same as figure 1, but for $h \equiv h_{2}$ scenario.
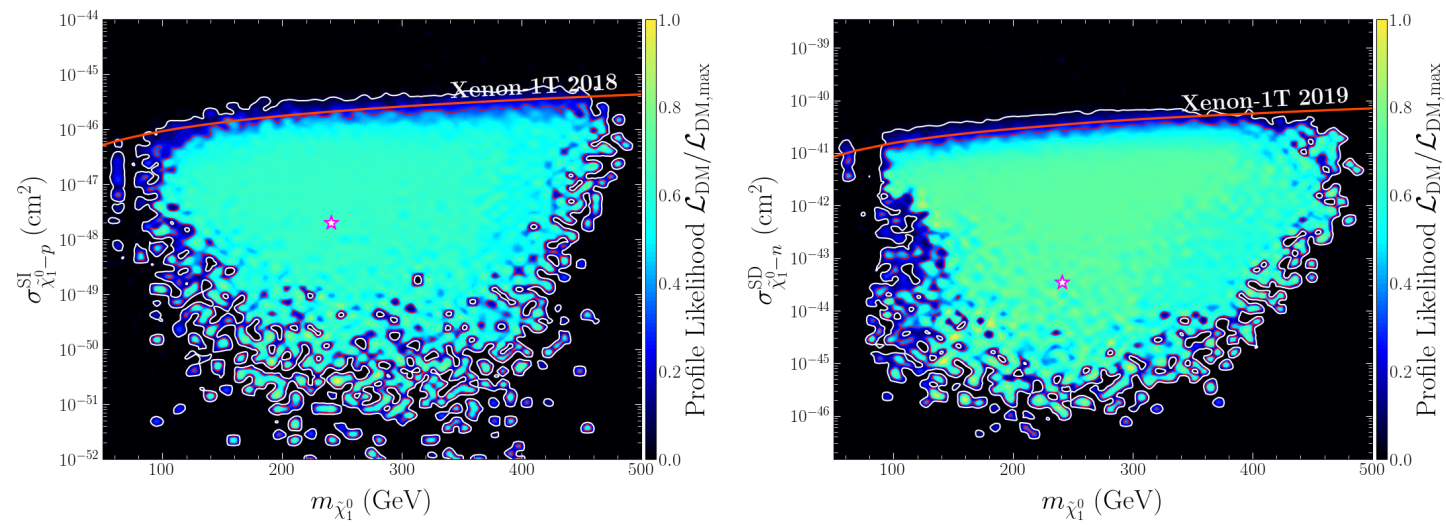

Figure 4. Same as figure 2, but for $h \equiv h_{2}$ scenario.

completely different from the PL of the total likelihood function $\mathcal{L}$ in eq. (3.1). Specifically, the $\chi^{2}$ of $\mathcal{L}$ is extremely dominated by the Higgs data fit, which contains 102 measurements in the code HiggsSignal-2.2.3 [64]. Thus, studying the distribution of $\mathcal{L}\left(\Theta_{A}, \Theta_{B}\right)$ is roughly equivalent to performing a Higgs data fit on the $\Theta_{A}-\Theta_{B}$ plane, which has nothing to do with DM physics. The other subtlety is that some of the scanned samples may be in serious conflict with the Higgs data or B-physics measurements, although they coincide well with the DM experiments. Since $\mathcal{L}$ strongly disfavors these samples, they should be neglected. Therefore, only the following samples are considered in studying $\mathcal{L}_{\mathrm{DM}}\left(\Theta_{A}, \Theta_{B}\right)$.

- Its $p$ value in the Higgs data fit is larger than 0.05, which implies that it coincides with the data at $95 \%$ confidence level.

- It is consistent with the extra Higgs search results implemented in the code HiggsBounds-5.3.2.

- It accounts for the measurement of $\operatorname{Br}\left(B \rightarrow X_{s} \gamma\right)$ and $\operatorname{Br}\left(B_{s}^{0} \rightarrow \mu^{+} \mu^{-}\right)$at $2 \sigma$ level [66]. 
Using the refined samples, different 2D PL maps are shown in figures 1 to 4 . Figure 1 plots the credible interval (CI) on $|\kappa|-\lambda$ and $\left|\mu_{\text {tot }}\right|-m_{\tilde{\chi}_{1}^{0}}$ plane for the $h \equiv h_{1}$ scenario. This plot indicates that the theory can explain the DM experiments quite well when $\lambda \lesssim 0.5$, $0.05 \lesssim|\kappa| \lesssim 0.5, m_{\tilde{\chi}_{1}^{0}} \gtrsim 140 \mathrm{GeV}$, and $\left|\mu_{\text {tot }}\right| \gtrsim 160 \mathrm{GeV}$. The best point locates at approximately $\lambda=0.05, \kappa=0.28, m_{\tilde{\chi}_{1}^{0}}=350 \mathrm{GeV}$, and $\mu_{\text {tot }}=450 \mathrm{GeV}$. Additionally, $2|\kappa| / \lambda$ can be much larger than 1 to keep $\tilde{\chi}_{1}^{0}$ Singlino-dominated, which is one distinct feature of the $\mu$ NMSSM. Figure 2 shows the $1 \sigma$ and $2 \sigma$ CIs on the $\sigma_{\tilde{\chi}_{1}^{0}-p}^{\mathrm{SI}}-m_{\tilde{\chi}_{1}^{0}}$ and $\sigma_{\tilde{\chi}_{1}^{0}-n}^{\mathrm{SD}}-m_{\tilde{\chi}_{1}^{0}}$ planes. This plot indicates that the SI and SD cross-sections can be as low as $10^{-51} \mathrm{~cm}^{2}$ and $10^{-46} \mathrm{~cm}^{2}$, respectively, over a broad mass range of $\tilde{\chi}_{1}^{0}$. This conclusion comes from the fact that $\lambda$ can be small and the ratio $m_{\tilde{\chi}_{1}^{0}} / \mu_{\text {tot }}$ in eqs. $(2.32)-(2.32)$ can be much less than 1. Figures 3 and 4 are similar to figures 1 and 2, except for the $h \equiv h_{2}$ scenario. Compared with the $h \equiv h_{1}$ scenario, this scenario has the following different characteristics.

- The CI on $|\kappa|-\lambda$ plane shrinks significantly.

- $m_{\tilde{\chi}_{1}^{0}}$ as low as $100 \mathrm{GeV}$ can explain the experiments.

- The best point locates at approximately $\kappa=0.2$ and $m_{\tilde{\chi}_{1}^{0}}=250 \mathrm{GeV}$.

In addition to the fact that the parameter space of the $h \equiv h_{2}$ scenario is relatively narrow to fit well with the Higgs data [34], another reason for the difference is that, since $h_{s}=h_{1}$ is light, a moderately light $\tilde{\chi}_{1}^{0}$ can make the annihilation $\tilde{\chi}_{1}^{0} \tilde{\chi}_{1}^{0} \rightarrow h_{s} A_{s}$ occur.

A detailed explanation of the PL figure, including how to plot it and understand it correctly, was presented in our previous works [10,60]. For the sake of brevity, it is not repeated here. Besides, the fact that the marginal posterior PDF and the PL differ significantly in their definitions is worth noting. Therefore, they are complementary to each other in describing the results of the present work.

\subsection{Constraints from LHC search for electroweakinos}

In the GNMSSM, the natural electroweak symmetry breaking tends to a Higgsino mass of several hundreds of $\mathrm{GeV}$. In this case, the LHC will produce Higgsino pair events copiously, and the Higgsino's property is strongly restricted by searching for multi-lepton signals. Up to now, experimental analyses in this aspect usually considered Wino pair production and provided the Wino mass bound as a function of $m_{\tilde{\chi}_{1}^{0}}$ in the simplified model. Recently, the ATLAS collaboration analyzed $139 \mathrm{fb}^{-1}$ proton-proton collision data collected at the LHC with $\sqrt{s}=13 \mathrm{TeV}$ and concluded that the LHC had already explored the region with the Wino mass up to approximately $700 \mathrm{GeV}$ and $m_{\tilde{\chi}_{1}^{0}}$ up to $300 \mathrm{GeV}$ [82-84]. These analyses were applied to the Higgsino pair production process in the present study by elaborate Monte Carlo simulations and it was found that they can effectively limit the Higgsino's property when $m_{\tilde{\chi}_{1}^{0}} \lesssim 100 \mathrm{GeV} .{ }^{5}$ As a specific application, the $h$-funnel region

\footnotetext{
${ }^{5}$ A similar conclusion was obtained as shown in figure 3 of [10], where the results on the upper panel of figure 8 in [85] are re-interpreted in terms of the Higgsino pair production process. The latter result was obtained by a combined analysis of the multi-lepton signal by the CMS collaboration. Its exclusion capability on the Wino mass versus DM mass plane is roughly identical to that of the analyses in [82-84].
} 


\begin{tabular}{|c|c|c|c|c|}
\hline & \multicolumn{3}{|c|}{$\mathrm{h} \equiv \mathrm{h}_{1}$ Scenario } & \multirow{2}{*}{$\frac{\mathrm{h} \equiv \mathrm{h}_{2} \text { Scenario }}{\text { Point IV }}$} \\
\hline & Point I & Point II & Point III & \\
\hline$\lambda$ & 0.026 & 0.227 & 0.038 & 0.077 \\
\hline$\kappa$ & 0.236 & 0.328 & -0.212 & 0.199 \\
\hline $\tan \beta$ & 9.750 & 6.698 & 9.454 & 8.092 \\
\hline$\mu(\mathrm{GeV})$ & 322.6 & 243.8 & 226.7 & 291.3 \\
\hline$\mu_{\text {tot }}(\mathrm{GeV})$ & 335.0 & 331.9 & 248.0 & 338.2 \\
\hline$A_{\kappa}(\mathrm{GeV})$ & -310.5 & -238.1 & 21.58 & -415.8 \\
\hline$A_{t}(\mathrm{GeV})$ & 4244 & 4112 & 2999 & 2959 \\
\hline$m_{h_{1}}(\mathrm{GeV})$ & 125.2 & 125.2 & 125.1 & 93.67 \\
\hline$m_{h_{2}}(\mathrm{GeV})$ & 134.8 & 203.9 & 233.3 & 125.2 \\
\hline$m_{h_{3}}(\mathrm{GeV})$ & 835.3 & 1273 & 794.6 & 1053 \\
\hline$m_{A_{s}}(\mathrm{GeV})$ & 326.9 & 310.6 & 86.62 & 388.4 \\
\hline$m_{\tilde{\chi}_{1}^{0}}(\mathrm{GeV})$ & 228.6 & 249.2 & 236.7 & 255.4 \\
\hline$m_{\tilde{\chi}_{2}^{0}}(\mathrm{GeV})$ & 343.5 & 343.2 & 254.5 & 386.7 \\
\hline$m_{\tilde{\chi}_{3}^{0}}(\mathrm{GeV})$ & 345.4 & 345.5 & 258.0 & 348.7 \\
\hline$m_{\tilde{\chi}_{1}^{ \pm}}(\mathrm{GeV})$ & 344.6 & 340.7 & 255.7 & 387.8 \\
\hline$V_{h}^{\mathrm{NSM}}, V_{h}^{\mathrm{SM}}, V_{h}^{\mathrm{S}}$ & $-0.20,-0.94,0.30$ & $-0.30,-0.95,-0.06$ & $-0.21,-0.98,0.02$ & $0.01,0.99,0.17$ \\
\hline$V_{h_{s}}^{\mathrm{NSM}}, V_{h_{s}}^{\mathrm{SM}}, V_{h_{s}}^{\mathrm{S}}$ & $-0.05,-0.29,-0.96$ & $-0.05,0.08,-0.99$ & $-0.02,-0.01,-0.99$ & $0.01,-0.17,0.99$ \\
\hline$N_{13}, N_{14}, N_{15}$ & $0.01,-0.02,0.99$ & $0.14,-0.21,0.97$ & $-0.17,-0.18,-0.97$ & $0.04,-0.07,0.99$ \\
\hline$N_{23}, N_{24}, N_{25}$ & $-0.71,0.71,0.02$ & $0.71,0.71,0.05$ & $-0.71,0.71,0.05$ & $-0.71,0.70,0.08$ \\
\hline$N_{33}, N_{34}, N_{35}$ & $0.71,0.71,0.01$ & $-0.69,0.68,0.25$ & $-0.69,-0.69,-0.25$ & $0.71,0.71,0.02$ \\
\hline$\Omega h^{2}$ & 0.125 & 0.125 & 0.131 & 0.124 \\
\hline$\sigma_{\tilde{\chi}_{1}^{0}-p}^{S I}\left(\mathrm{~cm}^{2}\right)$ & $2.83 \times 10^{-48}$ & $2.59 \times 10^{-47}$ & $1.01 \times 10^{-47}$ & $6.36 \times 10^{-48}$ \\
\hline$\sigma_{\tilde{\chi}_{1}^{0}-n}^{S D}\left(\mathrm{~cm}^{2}\right)$ & $2.47 \times 10^{-46}$ & $1.95 \times 10^{-41}$ & $5.49 \times 10^{-43}$ & $2.50 \times 10^{-44}$ \\
\hline Annihilation processes & $\begin{aligned} 71 \% \tilde{\chi}_{1}^{0} \tilde{\chi}_{1}^{0} & \rightarrow \mathrm{h}_{\mathrm{s}} \mathrm{A}_{\mathrm{s}} \\
20 \% \tilde{\chi}_{1}^{0} \tilde{\chi}_{1}^{0} & \rightarrow \mathrm{h} \mathrm{A} \mathrm{A}_{\mathrm{s}} \\
7.4 \% \tilde{\chi}_{1}^{0} \tilde{\chi}_{1}^{0} & \rightarrow \mathrm{h}_{\mathrm{s}} \mathrm{h}_{\mathrm{s}} \\
1.3 \% \tilde{\chi}_{1}^{0} \tilde{\chi}_{1}^{0} & \rightarrow \mathrm{h} \mathrm{h} \mathrm{h}_{\mathrm{s}} \\
\cdots & \end{aligned}$ & $\begin{array}{ll}34 \% & \tilde{\chi}_{1}^{0} \tilde{\chi}_{1}^{0} \rightarrow \mathrm{t} \overline{\mathrm{t}} \\
33 \% & \tilde{\chi}_{1}^{0} \tilde{\chi}_{1}^{0} \rightarrow \mathrm{h}_{\mathrm{s}} \mathrm{A}_{\mathrm{s}} \\
27 \% & \tilde{\chi}_{1}^{0} \tilde{\chi}_{1}^{0} \rightarrow \mathrm{h}_{\mathrm{s}} \mathrm{h}_{\mathrm{s}} \\
1.4 \% & \tilde{\chi}_{1}^{0} \tilde{\chi}_{1}^{0} \rightarrow \mathrm{h} \mathrm{A} \mathrm{A}_{\mathrm{s}} \\
1.1 \% & \tilde{\chi}_{1}^{0} \tilde{\chi}_{1}^{0} \rightarrow \mathrm{W}^{+} \mathrm{W}^{-} \\
& \ldots\end{array}$ & $\begin{array}{cc}5.4 \% & \tilde{\chi}_{2}^{0} \tilde{\chi}_{1}^{ \pm} \rightarrow \mathrm{d} \overline{\mathrm{u}} \\
5.4 \% & \tilde{\chi}_{2}^{0} \tilde{\chi}_{1}^{ \pm} \rightarrow \mathrm{s} \overline{\mathrm{c}} \\
4.0 \% & \tilde{\chi}_{2}^{0} \tilde{\chi}_{1}^{ \pm} \rightarrow \mathrm{b} \overline{\mathrm{t}} \\
4.0 \% & \tilde{\chi}_{1}^{0} \tilde{\chi}_{1}^{ \pm} \rightarrow \mathrm{s} \overline{\mathrm{c}} \\
4.0 \% & \tilde{\chi}_{1}^{0} \tilde{\chi}_{1}^{ \pm} \rightarrow \mathrm{d} \overline{\mathrm{u}} \\
\ldots\end{array}$ & $\begin{array}{l}96 \% \tilde{\chi}_{1}^{0} \tilde{\chi}_{1}^{0} \rightarrow \mathrm{h}_{\mathrm{s}} \mathrm{A}_{\mathrm{s}} \\
3.4 \% \tilde{\chi}_{1}^{0} \tilde{\chi}_{1}^{0} \rightarrow \mathrm{h}_{\mathrm{s}} \mathrm{h}_{\mathrm{s}}\end{array}$ \\
\hline$\tilde{\chi}_{2}^{0}$ Decay channels & $100 \% \quad \tilde{\chi}_{2}^{0} \rightarrow \tilde{\chi}_{1}^{0} \mathrm{Z}$ & $100 \% \quad \tilde{\chi}_{2}^{0} \rightarrow \tilde{\chi}_{1}^{0} \mathrm{Z}$ & $\begin{array}{ll}16 \% & \tilde{\chi}_{2}^{0} \rightarrow \tilde{\chi}_{1}^{0} \mathrm{~d} \overline{\mathrm{d}}(\mathrm{d}=\mathrm{d}, \mathrm{s}) \\
12 \% & \tilde{\chi}_{2}^{0} \rightarrow \tilde{\chi}_{1}^{0} \mathrm{u} \overline{\mathrm{u}}(\mathrm{u}=\mathrm{u}, \mathrm{c}) \\
10 \% & \tilde{\chi}_{2}^{0} \rightarrow \tilde{\chi}_{1}^{0} \mathrm{~b} \overline{\mathrm{b}} \\
7.4 \% & \tilde{\chi}_{2}^{0} \rightarrow \tilde{\chi}_{1}^{0} \nu \bar{\nu}\left(\nu=\nu_{\mathrm{e}}, \nu_{\mu}, \nu_{\tau}\right) \\
3.7 \% & \tilde{\chi}_{2}^{0} \rightarrow \tilde{\chi}_{1}^{0} \mathrm{l} \overline{\mathrm{l}}(\mathrm{l}=\mathrm{e}, \mu) \\
3.5 \% & \tilde{\chi}_{2}^{0} \rightarrow \tilde{\chi}_{1}^{0} \tau \bar{\tau}\end{array}$ & $\begin{aligned} 57 \% & \tilde{\chi}_{2}^{0} \rightarrow \tilde{\chi}_{1}^{0} \mathrm{Z} \\
43 \% & \tilde{\chi}_{2}^{0} \rightarrow \tilde{\chi}_{1}^{0} \mathrm{~h}_{\mathrm{s}}\end{aligned}$ \\
\hline$\tilde{\chi}_{3}^{0}$ Decay channels & $100 \% \quad \tilde{\chi}_{3}^{0} \rightarrow \tilde{\chi}_{1}^{0} \mathrm{Z}$ & $100 \% \quad \tilde{\chi}_{3}^{0} \rightarrow \tilde{\chi}_{1}^{0} \mathrm{Z}$ & $\begin{array}{ll}11 \% & \tilde{\chi}_{3}^{0} \rightarrow \tilde{\chi}_{1}^{0} \mathrm{~d} \overline{\mathrm{d}}(\mathrm{d}=\mathrm{d}, \mathrm{s}) \\
8.3 \% & \tilde{\chi}_{3}^{0} \rightarrow \tilde{\chi}_{1}^{0} \mathrm{u} \overline{\mathrm{u}} \\
7.9 \% & \tilde{\chi}_{3}^{0} \rightarrow \tilde{\chi}_{1}^{0} \mathrm{c} \overline{\mathrm{c}} \\
6.8 \% & \tilde{\chi}_{3}^{0} \rightarrow \tilde{\chi}_{1}^{0} \mathrm{~b} \overline{\mathrm{b}} \\
5.6 \% & \tilde{\chi}_{3}^{0} \rightarrow \tilde{\chi}_{3}^{0} \mathrm{~b} \overline{\mathrm{b}} \\
5.0 \% & \tilde{\chi}_{3}^{0} \rightarrow \tilde{\chi}_{1}^{0} \nu \bar{\nu}\left(\nu=\nu_{e}, \nu_{\mu}, \nu_{\tau}\right)\end{array}$ & $\begin{array}{l}99.7 \% \tilde{\chi}_{3}^{0} \rightarrow \tilde{\chi}_{1}^{0} \mathrm{Z} \\
0.3 \% \quad \tilde{\chi}_{3}^{0} \rightarrow \tilde{\chi}_{1}^{0} \mathrm{~h}_{\mathrm{s}}\end{array}$ \\
\hline$\tilde{\chi}_{1}^{ \pm}$Decay channels & $100 \% \tilde{\chi}_{1}^{ \pm} \rightarrow \tilde{\chi}_{1}^{0} \mathrm{~W}^{ \pm}$ & $100 \% \quad \tilde{\chi}_{1}^{ \pm} \rightarrow \tilde{\chi}_{1}^{0} \mathrm{~W}^{ \pm}$ & $\begin{array}{ll}33 \% & \tilde{\chi}_{2}^{ \pm} \rightarrow \tilde{\chi}_{1}^{0} \mathrm{u} \overline{\mathrm{d}}(\mathrm{u} \overline{\mathrm{d}}=\mathrm{u} \overline{\mathrm{d}}, \mathrm{c} \overline{\mathrm{s}}) \\
11 \% & \tilde{\chi}_{1}^{ \pm} \rightarrow \tilde{\chi}_{1}^{0} \nu \overline{\mathrm{l}}\left(\nu \overline{\mathrm{l}}=\nu_{\mathrm{e}} \overline{\mathrm{e}}, \nu_{\nu} \bar{\mu}, \nu_{\tau} \bar{\tau}\right)\end{array}$ & $100 \% \quad \tilde{\chi}_{1}^{ \pm} \rightarrow \tilde{\chi}_{1}^{0} \mathrm{~W}^{ \pm}$ \\
\hline
\end{tabular}

Table 4. Detailed information of four benchmark points that agree well with all considered experiments. Number before each annihilation process represents its fraction in contributing to total DM annihilation cross-section at freeze-out temperature. Number before each decay denotes its branching ratio. 


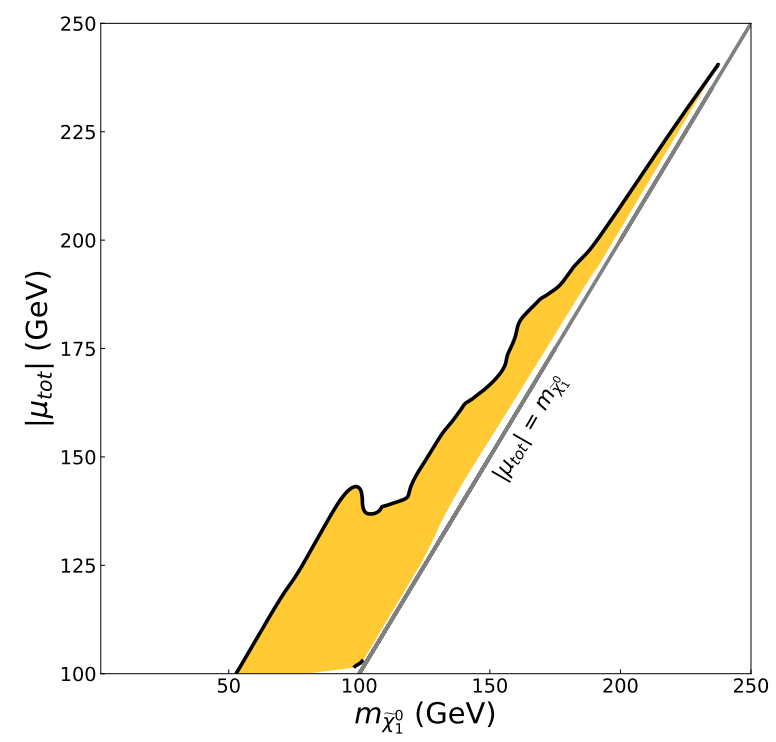

Figure 5. Constraint from latest ATLAS analysis of di-lepton signal at 13-TeV LHC, which is shown on $\left|\mu_{\text {tot }}\right|-m_{\tilde{\chi}_{1}^{0}}$ plane. Analysis is concentrated on compressed mass spectra [86] and excludes the yellow-shaded region at $95 \%$ confidence level when $\lambda=0.01$ and $\tan \beta=10$ are fixed.

in the $h \equiv h_{2}$ scenario has been excluded by the analyses. This result is consistent with our previous observation in [9] that the area has been ruled out by CMS's search for the multi-lepton signal at the $13-\mathrm{TeV}$ LHC with $35.9 \mathrm{fb}^{-1}$ data [85].

Above discussion reveals that the analyses in [82-85] have little restriction on the theory since $\tilde{\chi}_{1}^{0}$ in the GNMSSM is preferred heavier than $100 \mathrm{GeV}$. This fact motivates us to consider an experimental analysis that can detect high-DM-mass region [86]. The analysis aims to explore compressed mass spectra and utilizes the $139 \mathrm{fb}^{-1}$ data of the LHC. Events with missing transverse momentum and two same-flavor, oppositely charged, lowtransverse-momentum leptons are selected, and are further categorized by the presence of hadronic activity from initial-state radiation. The analysis was reproduced in the present work by using the simulation tools MadGraph5_aMC@NLO-2.6.6 [87, 88] to generate the parton-level events of the processes $p p \rightarrow \tilde{\chi}_{2}^{0} \tilde{\chi}_{3}^{0}(j), \tilde{\chi}_{2}^{0} \tilde{\chi}_{1}^{ \pm}(j), \tilde{\chi}_{3}^{0} \tilde{\chi}_{1}^{ \pm}(j)$, and $\tilde{\chi}_{1}^{ \pm} \tilde{\chi}_{1}^{\mp}(j)$; Pythia8.2 [89] for parton fragmentation and hadronization; Delphes-3.4.2 [90] for fast simulation of the performance of the ATLAS detector; and CheckMATE-2.0.26 [91-93] to implement the analysis' cut selections. The validation of the method is provided in our recent work [14].

In figure 5 , the excluded region is shown on the $\left|\mu_{\text {tot }}\right|-m_{\tilde{\chi}_{1}^{0}}$ plane. $\lambda=0.01, \tan \beta=10$, and $M_{1}=M_{2}=3 \mathrm{TeV}$ are fixed, and the procedure depicted in [59] is followed to plot the figure. The result indicates that the analysis can explore $m_{\tilde{\chi}_{1}^{0}}$ up to $230 \mathrm{GeV}$ and significantly impact the co-annihilation mechanism. In addition, it is noticeable that the excluded region in the present work is broader than that on the upper panel of figure 14 in [86]. This is because $\tilde{\chi}_{1}^{0}$ is Singlino-dominated in this work, instead of Higgsino-dominated in [86], so more processes contribute to the signal.

To fully demonstrate the Singlino-dominated DM scenario's characteristics, the details of four benchmark points are provided in table 4 . The first three points belong to the $h \equiv h_{1}$ 
scenario and the last one is from the $h \equiv h_{2}$ scenario. They all predict $m_{\tilde{\chi}_{1}^{0}} \gtrsim 230 \mathrm{GeV}$ and thus are difficult to detect at the LHC. In addition to the characteristics listed in the table, the following features merit emphasizing.

- If the mass splitting between $\tilde{\chi}_{i}^{0}(i=2,3)$ and $\tilde{\chi}_{1}^{0}$ is larger than any of the neutral Higgs boson masses, $\tilde{\chi}_{i}^{0}$ will decay with sizable branching ratios into the Higgs bosons. Otherwise, it will decay into a real or virtual $Z$ boson.

- Although the process $\tilde{\chi}_{1}^{0} \tilde{\chi}_{1}^{0} \rightarrow h_{s} A_{s}$ is mainly responsible for the abundance in most cases, its role diminishes when $m_{h_{s}}+m_{A_{s}}$ approaches $2 m_{\tilde{\chi}_{1}^{0}}$ from below. This situation is demonstrated by Points 1 and 2 .

- Since $V_{h}^{S}=0.3$ is sizable, $\tilde{\chi}_{1}^{0} \tilde{\chi}_{1}^{0} \rightarrow h A_{s}$ for Point 1 also plays a crucial role in determining the abundance.

- Since $\lambda=0.227$ is sizable, $\sigma_{\tilde{\chi}_{1}^{0}-p}^{\mathrm{SI}}$ and $\sigma_{\tilde{\chi}_{1}^{0}-n}^{\mathrm{SD}}$ for Point 2 are relatively large.

- Since $m_{\tilde{\chi}_{1}^{0}} / \mu_{\text {tot }}=0.95$ approaches $1, \sigma_{\tilde{\chi}_{1}^{0}-p}^{\mathrm{SI}}$ and $\sigma_{\tilde{\chi}_{1}^{0}-n}^{\mathrm{SD}}$ for Point 3 are relatively large even though $\lambda$ is small.

- Point 4 predicts a significant cancellation between the $h$ - and $h_{s}$-mediated contributions to $\sigma_{\tilde{\chi}_{1}^{0}-p}^{\mathrm{SI}}$.

\section{Conclusions}

Motivated by the increasingly tight limitation of the direct detection experiments on traditional neutralino DM in the natural MSSM and $Z_{3}$-NMSSM, the $Z_{3}$-NMSSM is extended in the present work by adding a $\mu \hat{H}_{u} \cdot \hat{H}_{d}$ term in its superpotential, and whether the Singlino-dominated neutralino can act as a feasible DM candidate is studied. Different from the $Z_{3}$-NMSSM, the extended theory describes the neutralino's property by four independent parameters: $\lambda, \mu_{\text {tot }}, m_{\tilde{\chi}_{1}^{0}}$, and $\kappa$. The first three parameters strongly influence the DM-nucleon scattering rate, while $\kappa$ usually only slightly affects the scattering. This characteristic implies that singlet-dominated particles may form a secluded DM sector. Under such a theoretical structure, the Singlino-dominated neutralino achieves the correct abundance by annihilating into a pair of singlet-dominated Higgs bosons by adjusting $\kappa$ 's value. Its scattering with nucleons is suppressed when $\lambda v / \mu_{\text {tot }}$ is small.

Our speculations are verified by numerical results. Specifically, a likelihood function containing the current experimental and theoretical situations of DM physics, Higgs physics, and B physics was constructed, which was then utilized to guide sophisticated scans of the theory's parameter space by the nest sampling algorithm. The scanned samples were analyzed by several statistical quantities, such as the marginal posterior PDF and profile likelihood, to reveal the underlying physics. The results show that, in the theory's natural space for electroweak symmetry breaking, the DM obtains the correct abundance by $\tilde{\chi}_{1}^{0} \tilde{\chi}_{1}^{0} \rightarrow h_{s} A_{s}$ in most cases, and the SI and SD cross-sections may be as low as $10^{-51} \mathrm{~cm}^{2}$ and $10^{-46} \mathrm{~cm}^{2}$, respectively. Furthermore, the LHC search for electroweakinos restricts the theory very weakly. 
It is emphasized herein that the $Z_{3}$-NMSSM differs significantly from the extended theory in at least three aspects. First, $m_{\tilde{\chi}_{1}^{0}}$ and $\kappa$ are no longer independent once one takes $\lambda$ and $\mu_{\text {tot }}$ as inputs to study the DM's property. Second, $|\kappa|$ must be less than $\lambda / 2$ to keep the lightest neutralino Singlino-dominated. Its magnitude should be small after considering the tight constraints from the direct detection experiments. Third, due to the absence of the $\mu$-induced contributions to the Higgs squared mass, the singlet-dominated Higgs particles tend to be more massive. Combined with these facts, the DM cannot obtain the correct abundance by $\tilde{\chi}_{1}^{0} \tilde{\chi}_{1}^{0} \rightarrow h_{s} A_{s}$. Instead, it must co-annihilate with the Higgsinos to keep consistent with the DM experimental results, which corresponds to the correlated parameter space $\lambda \simeq 2|\kappa|$ with $\lambda \lesssim 0.1$. The Bayesian evidence of the two theories was compared and it was found that their Jeffrey's-scale value is 8.05. This result implies that the considered experiments strongly prefer the extended theory. Since it resurrects the $Z_{3}$-NMSSM's broad parameter space that has been experimentally excluded, the extended theory is attractive and worthy of a careful study.

\section{Acknowledgments}

We thank Di Zhang for useful discussions about the LHC search for supersymmetry. This work is supported by the National Natural Science Foundation of China (NNSFC) under Grant Nos. 11575053 and 12075076.

Open Access. This article is distributed under the terms of the Creative Commons Attribution License (CC-BY 4.0), which permits any use, distribution and reproduction in any medium, provided the original author(s) and source are credited.

\section{References}

[1] Planck collaboration, Planck 2015 results. XIII. Cosmological parameters, Astron. Astrophys. 594 (2016) A13 [arXiv:1502.01589] [INSPIRE].

[2] Planck collaboration, Planck 2018 results. VI. Cosmological parameters, Astron. Astrophys. 641 (2020) A6 [arXiv: 1807.06209] [INSPIRE].

[3] K. Griest and M. Kamionkowski, Supersymmetric dark matter, Phys. Rept. 333 (2000) 167 [INSPIRE].

[4] G. Bertone, D. Hooper and J. Silk, Particle dark matter: Evidence, candidates and constraints, Phys. Rept. 405 (2005) 279 [hep-ph/0404175] [INSPIRE].

[5] S. Baum, M. Carena, N.R. Shah and C.E.M. Wagner, Higgs portals for thermal Dark Matter. EFT perspectives and the NMSSM, JHEP 04 (2018) 069 [arXiv:1712.09873] [INSPIRE].

[6] XENON collaboration, Dark Matter Search Results from a One Ton-Year Exposure of XENON1T, Phys. Rev. Lett. 121 (2018) 111302 [arXiv:1805.12562] [INSPIRE].

[7] PandaX-II collaboration, Results of dark matter search using the full PandaX-II exposure, Chin. Phys. C 44 (2020) 125001 [arXiv:2007.15469] [INSPIRE].

[8] PandaX-II collaboration, Dark Matter Results From 54-Ton-Day Exposure of PandaX-II Experiment, Phys. Rev. Lett. 119 (2017) 181302 [arXiv:1708.06917] [INSPIRE]. 
[9] J. Cao, Y. He, L. Shang, Y. Zhang and P. Zhu, Current status of a natural NMSSM in light of LHC 13 TeV data and XENON-1T results, Phys. Rev. D 99 (2019) 075020 [arXiv: 1810.09143] [INSPIRE].

[10] J. Cao, L. Meng, Y. Yue, H. Zhou and P. Zhu, Suppressing the scattering of WIMP dark matter and nucleons in supersymmetric theories, Phys. Rev. D 101 (2020) 075003 [arXiv: 1910.14317] [INSPIRE].

[11] E. Bagnaschi et al., Likelihood Analysis of the pMSSM11 in Light of LHC 13-TeV Data, Eur. Phys. J. C 78 (2018) 256 [arXiv:1710.11091] [InSPIRE].

[12] U. Ellwanger, C. Hugonie and A.M. Teixeira, The Next-to-Minimal Supersymmetric Standard Model, Phys. Rept. 496 (2010) 1 [arXiv:0910.1785] [InSPIRE].

[13] J. Cao, Y. He, L. Shang, W. Su, P. Wu and Y. Zhang, Strong constraints of LUX-2016 results on the natural NMSSM, JHEP 10 (2016) 136 [arXiv:1609.00204] [INSPIRE].

[14] H. Zhou, J. Cao, J. Lian and D. Zhang, Singlino-dominated dark matter in $Z_{3}$-NMSSM, arXiv:2102.05309 [INSPIRE].

[15] J. Cao, L. Shang, P. Wu, J.M. Yang and Y. Zhang, Interpreting the galactic center gamma-ray excess in the NMSSM, JHEP 10 (2015) 030 [arXiv:1506.06471] [INSPIRE].

[16] M. Pospelov, A. Ritz and M.B. Voloshin, Secluded WIMP Dark Matter, Phys. Lett. B 662 (2008) 53 [arXiv:0711.4866] [INSPIRE].

[17] D. Das, U. Ellwanger and A.M. Teixeira, Modified Signals for Supersymmetry in the NMSSM with a Singlino-like LSP, JHEP 04 (2012) 067 [arXiv:1202.5244] [INSPIRE].

[18] U. Ellwanger and A.M. Teixeira, NMSSM with a singlino LSP: possible challenges for searches for supersymmetry at the LHC, JHEP 10 (2014) 113 [arXiv:1406.7221] [INSPIRE].

[19] U. Ellwanger, Present Status and Future Tests of the Higgsino-Singlino Sector in the NMSSM, JHEP 02 (2017) 051 [arXiv: 1612.06574] [inSPIRE].

[20] U. Ellwanger and C. Hugonie, The higgsino-singlino sector of the NMSSM: combined constraints from dark matter and the LHC, Eur. Phys. J. C 78 (2018) 735 [arXiv: 1806.09478] [INSPIRE].

[21] Q.-F. Xiang, X.-J. Bi, P.-F. Yin and Z.-H. Yu, Searching for Singlino-Higgsino Dark Matter in the NMSSM, Phys. Rev. D 94 (2016) 055031 [arXiv:1606.02149] [INSPIRE].

[22] W. Abdallah, A. Chatterjee and A. Datta, Revisiting singlino dark matter of the natural $Z_{3}$-symmetric NMSSM in the light of LHC, JHEP 09 (2019) 095 [arXiv:1907.06270] [INSPIRE].

[23] M. Guchait and A. Roy, Light Singlino Dark Matter at the LHC, Phys. Rev. D 102 (2020) 075023 [arXiv: 2005.05190] [INSPIRE].

[24] H.M. Lee et al., A unique $\mathbb{Z}_{4}^{R}$ symmetry for the MSSM, Phys. Lett. B 694 (2011) 491 [arXiv: 1009.0905] [INSPIRE].

[25] H.M. Lee et al., Discrete $R$ symmetries for the MSSM and its singlet extensions, Nucl. Phys. $B 850$ (2011) 1 [arXiv:1102.3595] [INSPIRE].

[26] G.G. Ross and K. Schmidt-Hoberg, The Fine-Tuning of the Generalised NMSSM, Nucl. Phys. B 862 (2012) 710 [arXiv: 1108.1284] [InSPIRE].

[27] G.G. Ross, K. Schmidt-Hoberg and F. Staub, The Generalised NMSSM at One Loop: Fine Tuning and Phenomenology, JHEP 08 (2012) 074 [arXiv:1205.1509] [INSPIRE]. 
[28] S. Ferrara, R. Kallosh, A. Linde, A. Marrani and A. Van Proeyen, Jordan Frame Supergravity and Inflation in NMSSM, Phys. Rev. D 82 (2010) 045003 [arXiv: 1004.0712] [INSPIRE].

[29] S. Ferrara, R. Kallosh, A. Linde, A. Marrani and A. Van Proeyen, Superconformal Symmetry, NMSSM, and Inflation, Phys. Rev. D 83 (2011) 025008 [arXiv:1008.2942] [INSPIRE].

[30] M.B. Einhorn and D.R.T. Jones, Inflation with Non-minimal Gravitational Couplings in Supergravity, JHEP 03 (2010) 026 [arXiv:0912.2718] [INSPIRE].

[31] W.G. Hollik, S. Liebler, G. Moortgat-Pick, S. Paßehr and G. Weiglein, Phenomenology of the inflation-inspired NMSSM at the electroweak scale, Eur. Phys. J. C 79 (2019) 75 [arXiv: 1809.07371] [INSPIRE].

[32] D.J. Miller, R. Nevzorov and P.M. Zerwas, The Higgs sector of the next-to-minimal supersymmetric standard model, Nucl. Phys. B 681 (2004) 3 [hep-ph/0304049] [INSPIRE].

[33] W.G. Hollik, C. Li, G. Moortgat-Pick and S. Paasch, Phenomenology of a Supersymmetric Model Inspired by Inflation, Eur. Phys. J. C 81 (2021) 141 [arXiv:2004.14852] [INSPIRE].

[34] J.-J. Cao, Z.-X. Heng, J.M. Yang, Y.-M. Zhang and J.-Y. Zhu, A SM-like Higgs near $125 \mathrm{GeV}$ in low energy SUSY: a comparative study for MSSM and NMSSM, JHEP 03 (2012) 086 [arXiv: 1202.5821] [INSPIRE].

[35] L.J. Hall, D. Pinner and J.T. Ruderman, A Natural SUSY Higgs Near 126 GeV, JHEP 04 (2012) 131 [arXiv:1112.2703] [INSPIRE].

[36] U. Ellwanger, A Higgs boson near $125 \mathrm{GeV}$ with enhanced di-photon signal in the NMSSM, JHEP 03 (2012) 044 [arXiv: 1112.3548] [INSPIRE].

[37] S. Baum, N.R. Shah and K. Freese, The NMSSM is within Reach of the LHC: Mass Correlations 83 Decay Signatures, JHEP 04 (2019) 011 [arXiv: 1901.02332] [INSPIRE].

[38] M. Carena, I. Low, N.R. Shah and C.E.M. Wagner, Impersonating the Standard Model Higgs Boson: Alignment without Decoupling, JHEP 04 (2014) 015 [arXiv:1310.02248].

[39] M. Carena, H.E. Haber, I. Low, N.R. Shah and C.E.M. Wagner, Alignment limit of the NMSSM Higgs sector, Phys. Rev. D 93 (2016) 035013 [arXiv:1510.09137] [InSPIRE].

[40] P. Huang and C.E.M. Wagner, Blind Spots for neutralino Dark Matter in the MSSM with an intermediate $m_{A}$, Phys. Rev. D 90 (2014) 015018 [arXiv:1404.0392] [InSPIRE].

[41] ATLAS collaboration, Search for heavy Higgs bosons decaying into two tau leptons with the ATLAS detector using pp collisions at $\sqrt{s}=13 \mathrm{TeV}$, Phys. Rev. Lett. 125 (2020) 051801 [arXiv: 2002.12223] [INSPIRE].

[42] C. Cheung, M. Papucci, D. Sanford, N.R. Shah and K.M. Zurek, NMSSM Interpretation of the Galactic Center Excess, Phys. Rev. D 90 (2014) 075011 [arXiv: 1406.6372] [InSPIRE].

[43] M. Badziak, M. Olechowski and P. Szczerbiak, Blind spots for neutralino dark matter in the NMSSM, JHEP 03 (2016) 179 [arXiv: 1512.02472] [INSPIRE].

[44] M. Badziak, M. Olechowski and P. Szczerbiak, Spin-dependent constraints on blind spots for thermal singlino-higgsino dark matter with(out) light singlets, JHEP 07 (2017) 050 [arXiv: 1705.00227] [INSPIRE].

[45] K. Griest and D. Seckel, Three exceptions in the calculation of relic abundances, Phys. Rev. D 43 (1991) 3191 [INSPIRE].

[46] M.J. Baker et al., The Coannihilation Codex, JHEP 12 (2015) 120 [arXiv:1510.03434] [INSPIRE]. 
[47] K. Griest, M. Kamionkowski and M.S. Turner, Supersymmetric Dark Matter Above the $W$ Mass, Phys. Rev. D 41 (1990) 3565 [InSPIRE].

[48] M. Badziak, M. Olechowski and P. Szczerbiak, Blind spots for neutralinos in NMSSM with light singlet scalar, PoS PLANCK2015 (2015) 130 [arXiv:1601.00768] [INSPIRE].

[49] A. Pierce, N.R. Shah and K. Freese, Neutralino Dark Matter with Light Staus, arXiv:1309.7351 [INSPIRE].

[50] M. Drees and M. Nojiri, Neutralino-nucleon scattering revisited, Phys. Rev. D 48 (1993) 3483 [hep-ph/9307208] [INSPIRE].

[51] M. Drees and M.M. Nojiri, New contributions to coherent neutralino-nucleus scattering, Phys. Rev. D 47 (1993) 4226 [hep-ph/9210272] [INSPIRE].

[52] G. Bélanger, F. Boudjema, A. Pukhov and A. Semenov, Dark matter direct detection rate in a generic model with MicrOMEGAs 2.2, Comput. Phys. Commun. 180 (2009) 747 [arXiv:0803.2360] [INSPIRE].

[53] J.M. Alarcon, J. Martin Camalich and J.A. Oller, The chiral representation of the $\pi N$ scattering amplitude and the pion-nucleon sigma term, Phys. Rev. D 85 (2012) 051503 [arXiv:1110.3797] [INSPIRE].

[54] J.M. Alarcon, L.S. Geng, J. Martin Camalich and J.A. Oller, The strangeness content of the nucleon from effective field theory and phenomenology, Phys. Lett. B $\mathbf{7 3 0}$ (2014) 342 [arXiv: 1209.2870] [INSPIRE].

[55] G. Bélanger, F. Boudjema, C. Hugonie, A. Pukhov and A. Semenov, Relic density of dark matter in the NMSSM, JCAP 09 (2005) 001 [hep-ph/0505142] [INSPIRE].

[56] F. Feroz, M.P. Hobson and M. Bridges, MultiNest: an efficient and robust Bayesian inference tool for cosmology and particle physics, Mon. Not. Roy. Astron. Soc. 398 (2009) 1601 [arXiv:0809.3437] [INSPIRE].

[57] F. Feroz, M.P. Hobson, E. Cameron and A.N. Pettitt, Importance Nested Sampling and the MultiNest Algorithm, Open J. Astrophys. 2 (2019) 10 [arXiv:1306.2144] [INSPIRE].

[58] A. Fowlie and M.H. Bardsley, Superplot: a graphical interface for plotting and analysing MultiNest output, Eur. Phys. J. Plus 131 (2016) 391 [arXiv:1603.00555] [InSPIRE].

[59] J. Cao, J. Li, Y. Pan, L. Shang, Y. Yue and D. Zhang, Bayesian analysis of sneutrino dark matter in the NMSSM with a type-I seesaw mechanism, Phys. Rev. D 99 (2019) 115033 [arXiv: 1807.03762] [INSPIRE].

[60] J. Cao, Y. He, Y. Pan, Y. Yue, H. Zhou and P. Zhu, Impact of leptonic unitarity and dark matter direct detection experiments on the NMSSM with inverse seesaw mechanism, JHEP 12 (2020) 023 [arXiv: 1903.01124] [INSPIRE].

[61] XENON collaboration, Constraining the spin-dependent WIMP-nucleon cross sections with XENON1T, Phys. Rev. Lett. 122 (2019) 141301 [arXiv:1902.03234] [INSPIRE].

[62] Fermi-LAT data analysis, https://fermi.gsfc.nasa.gov/ssc/data/analysis/scitools/overview.html.

[63] Fermi-LAT collaboration, Searching for Dark Matter Annihilation from Milky Way Dwarf Spheroidal Galaxies with Six Years of Fermi Large Area Telescope Data, Phys. Rev. Lett. 115 (2015) 231301 [arXiv:1503.02641] [INSPIRE]. 
[64] P. Bechtle, S. Heinemeyer, O. Stål, T. Stefaniak and G. Weiglein, Probing the Standard Model with Higgs signal rates from the Tevatron, the LHC and a future ILC, JHEP 11 (2014) 039 [arXiv:1403.1582] [INSPIRE].

[65] P. Bechtle, S. Heinemeyer, O. Stål, T. Stefaniak and G. Weiglein, Applying Exclusion Likelihoods from LHC Searches to Extended Higgs Sectors, Eur. Phys. J. C 75 (2015) 421 [arXiv: 1507.06706] [INSPIRE].

[66] Particle Data Group collaboration, Review of Particle Physics, Phys. Rev. D 98 (2018) 030001 [INSPIRE].

[67] F. Staub, SARAH, arXiv:0806.0538 [inSPIRE].

[68] F. Staub, SARAH 3.2: Dirac Gauginos, UFO output, and more, Comput. Phys. Commun. 184 (2013) 1792 [arXiv: 1207.0906] [INSPIRE].

[69] F. Staub, SARAH 4: A tool for (not only SUSY) model builders, Comput. Phys. Commun. 185 (2014) 1773 [arXiv: 1309.7223] [INSPIRE].

[70] F. Staub, Exploring new models in all detail with SARAH, Adv. High Energy Phys. 2015 (2015) 840780 [arXiv:1503.04200] [INSPIRE].

[71] W. Porod, SPheno, a program for calculating supersymmetric spectra, SUSY particle decays and SUSY particle production at $e^{+} e^{-}$colliders, Comput. Phys. Commun. 153 (2003) 275 [hep-ph/0301101] [INSPIRE].

[72] W. Porod and F. Staub, SPheno 3.1: Extensions including flavour, CP-phases and models beyond the MSSM, Comput. Phys. Commun. 183 (2012) 2458 [arXiv:1104.1573] [InSPIRE].

[73] W. Porod, F. Staub and A. Vicente, A Flavor Kit for BSM models, Eur. Phys. J. C 74 (2014) 2992 [arXiv: 1405.1434] [INSPIRE].

[74] G. Bélanger, F. Boudjema, A. Pukhov and A. Semenov, MicrOMEGAs: A program for calculating the relic density in the MSSM, Comput. Phys. Commun. 149 (2002) 103 [hep-ph/0112278] [INSPIRE].

[75] G. Bélanger, F. Boudjema, A. Pukhov and A. Semenov, MicrOMEGAs 2.0: A program to calculate the relic density of dark matter in a generic model, Comput. Phys. Commun. 176 (2007) 367 [hep-ph/0607059] [INSPIRE].

[76] G. Bélanger, F. Boudjema, A. Pukhov and A. Semenov, MicrOMEGAs: A tool for dark matter studies, Nuovo Cim. C 033N2 (2010) 111 [arXiv:1005.4133] [INSPIRE].

[77] G. Bélanger, F. Boudjema, A. Pukhov and A. Semenov, MicrOMEGAs_3: A program for calculating dark matter observables, Comput. Phys. Commun. 185 (2014) 960 [arXiv: 1305.0237] [INSPIRE].

[78] D. Barducci et al., Collider limits on new physics within MicrOMEGAs_4.3, Comput. Phys. Commun. 222 (2018) 327 [arXiv:1606.03834] [INSPIRE].

[79] P. Gregory, Bayesian Logical Data Analysis for the Physical Sciences, Cambridge University Press, (2005).

[80] H. Jeffreys, The Theory of Probability, 3rd edition, Oxford University Press (1961), p. 432.

[81] F. Feroz, B.C. Allanach, M. Hobson, S.S. AbdusSalam, R. Trotta and A.M. Weber, Bayesian Selection of sign $\mu$ within $m S U G R A$ in Global Fits Including WMAP5 Results, JHEP 10 (2008) 064 [arXiv:0807.4512] [INSPIRE]. 
[82] ATLAS collaboration, Search for electroweak production of charginos and sleptons decaying into final states with two leptons and missing transverse momentum in $\sqrt{s}=13 \mathrm{TeV} \mathrm{pp}$ collisions using the ATLAS detector, Eur. Phys. J. C 80 (2020) 123 [arXiv:1908.08215] [INSPIRE].

[83] ATLAS collaboration, Search for chargino-neutralino production with mass splittings near the electroweak scale in three-lepton final states in $\sqrt{s}=13 \mathrm{TeV}$ pp collisions with the ATLAS detector, Phys. Rev. D 101 (2020) 072001 [arXiv:1912.08479] [INSPIRE].

[84] ATLAS collaboration, Search for chargino-neutralino pair production in final states with three leptons and missing transverse momentum in $\sqrt{s}=13 \mathrm{TeV} p$-p collisions with the ATLAS detector, Tech. Rep. ATLAS-CONF-2020-015, CERN, Geneva (Jun, 2020).

[85] CMS collaboration, Combined search for electroweak production of charginos and neutralinos in proton-proton collisions at $\sqrt{s}=13 \mathrm{TeV}$, JHEP 03 (2018) 160 [arXiv:1801.03957] [INSPIRE].

[86] ATLAS collaboration, Searches for electroweak production of supersymmetric particles with compressed mass spectra in $\sqrt{s}=13 \mathrm{TeV}$ pp collisions with the ATLAS detector, Phys. Rev. D 101 (2020) 052005 [arXiv:1911.12606] [InSPIRE].

[87] J. Alwall et al., The automated computation of tree-level and next-to-leading order differential cross sections, and their matching to parton shower simulations, JHEP 07 (2014) 079 [arXiv: 1405.0301] [INSPIRE].

[88] J. Alwall, M. Herquet, F. Maltoni, O. Mattelaer and T. Stelzer, MadGraph 5: Going Beyond, JHEP 06 (2011) 128 [arXiv:1106.0522] [InSPIRE].

[89] T. Sjöstrand, S. Mrenna and P.Z. Skands, PYTHIA 6.4 Physics and Manual, JHEP 05 (2006) 026 [hep-ph/0603175] [INSPIRE].

[90] DELPHES 3 collaboration, DELPHES 3, A modular framework for fast simulation of a generic collider experiment, JHEP 02 (2014) 057 [arXiv: 1307.6346] [INSPIRE].

[91] D. Dercks, N. Desai, J.S. Kim, K. Rolbiecki, J. Tattersall and T. Weber, CheckMATE 2: From the model to the limit, Comput. Phys. Commun. 221 (2017) 383 [arXiv:1611.09856] [INSPIRE].

[92] J.S. Kim, D. Schmeier, J. Tattersall and K. Rolbiecki, A framework to create customised LHC analyses within CheckMATE, Comput. Phys. Commun. 196 (2015) 535 [arXiv: 1503.01123] [INSPIRE].

[93] M. Drees, H. Dreiner, D. Schmeier, J. Tattersall and J.S. Kim, CheckMATE: Confronting your Favourite New Physics Model with LHC Data, Comput. Phys. Commun. 187 (2015) 227 [arXiv: 1312.2591] [INSPIRE]. 